\title{
AN APPROACH TO MODEL OPTIMIZATION OF MAN- UFACTURING OF EMITTER-COUPLED LOGIC
}

\author{
E.L. Pankratov ${ }^{1,3}$, E.A. Bulaeva ${ }^{1,2}$ \\ ${ }^{l}$ Nizhny Novgorod State University, 23 Gagarin avenue, Nizhny Novgorod, \\ 603950, Russia \\ ${ }^{2}$ Nizhny Novgorod State University of Architecture and Civil Engineering, 65 \\ Il'insky street, Nizhny Novgorod, 603950, Russia \\ ${ }^{3}$ Nizhny Novgorod Academy of the Ministry of Internal Affairs of Russia, 3 Ankudi- \\ novskoe Shosse, Nizhny Novgorod, 603950, Russia
}

\begin{abstract}
In this paper we consider an approach to optimization manufacturing elements of emitter-coupled logic. The optimization gives us possibility to decrease dimensions of these elements. The technological part based on manufacture a heterostructure with required configuration, doping by diffusion or ion implantation of required areas of heterostructure and optimization of annealing of dopant and/or radiation defects. The modelling part based on modified method of functional corrections, which using without crosslinking of solutions on interfaces between layers of heterostructure.
\end{abstract}

\section{KEYWORDS}

analytical approach for modelling; emitter-coupled logic; optimization of manufacturing; decreasing of dimensions; de-creasing of overheats

\section{INTRODUCTION}

Intensive development of electronic technique leads to increasing of performance of elements of integrated circuits ant to increasing of degree of their integration ( $p$ - $n$-junctions, bipolar and fieldeffect transistors, thyristors, ...) [1-6]. Increasing of the performance could be obtain by development of new and optimization of existing technological processes. Another way to increase the performance in determination of materials with higher speed of transport of charge carriers [710]. Increasing of degree of integration of elements of integrated circuits leads to necessity to decrease dimensions of the elements. To decrease the dimensions it could be used different approaches. Two of them are laser and microwave types of annealing [11-13]. Using this types of annealing leads to generation of inhomogenous distribution of temperature. The inhomogeneity leads to decreasing of dimensions of elements of integrated circuits due to Arrhenius law. Another way to decrease the dimensions is doping of epitaxial layers of heterostructures by diffusion and ion implantation [14-17]. However in this case it is practicably to optimize annealing of dopant and/or radiation defects [17-24]. It is known, that using radiation processing of materials of heterostructure and homogeneous samples leads to changing of distributions of concentrations of dopants in them [25].

In this paper as a development of works [17-24] we consider an approach to manufacture more compact elements of emitter-coupled logic. To illustrate the approach we consider a heterostructure, which is illustrated on Fig. 1. The heterostructure consist of a substrate and three epitaxial 
layers. Some sections have been manufactured in the epitaxial layers such as it shown on the figure. After finishing of the nearest to the substrate epitaxial layer we consider doping of the sections of the layer by diffusion or ion implantation. The sections will have role of collectors during functioning of the manufacturing device. Farther we consider annealing of dopant and/or radiation defects. After finishing this annealing we consider two another epitaxial layers with appropriate sections (see Fig. 1). After finishing of manufacturing of each epitaxial layer all sections of the last layer have been doped by diffusion or ion implantation. Farther we consider microwave annealing of dopant and/or radiation defects. Frequency of electro-magnetic radiation should be so, that thickness of scin-layer should be larger, than thickness of external epitaxial layer and smaller, than sum of thicknesses of external and average epitaxial layers. Sections of the average and external epitaxial layers will have roles of bases and emitter, respectively, during functioning of the considered device. Main aim of the present paper is analysis of dynamics of redistribution of infused and implanted dopants and/or radiation defects during annealing.

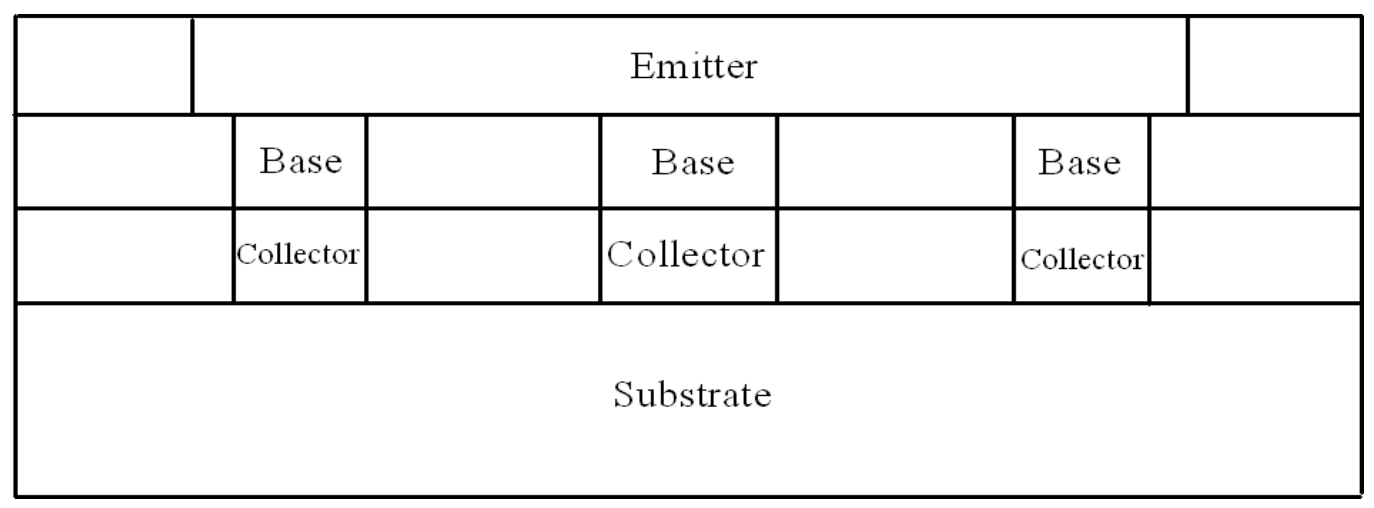

Fig. 1. Heterostructure with four layers, which consist of a substrate and three epitaxial layer with sections, manufactured by using another materials

\section{Method OF Solution}

To solve our aims we determine spatio-temporal distribution of concentration of dopant. We determine the required distribution by solving the second Fick's law [1,14 -17]

$$
\frac{\partial C(x, y, z, t)}{\partial t}=\frac{\partial}{\partial x}\left[D \frac{\partial C(x, y, z, t)}{\partial x}\right]+\frac{\partial}{\partial y}\left[D \frac{\partial C(x, y, z, t)}{\partial y}\right]+\frac{\partial}{\partial z}\left[D \frac{\partial C(x, y, z, t)}{\partial z}\right]
$$

with initial and boundary conditions

$$
\begin{gathered}
C(x, y, z, 0)=f_{C}(x, y, z),\left.\frac{\partial C(x, y, z, t)}{\partial x}\right|_{x=0}=0,\left.\frac{\partial C(x, y, z, t)}{\partial x}\right|_{x=L_{x}}=0,\left.\frac{\partial C(x, y, z, t)}{\partial y}\right|_{y=0}=0, \\
\left.\frac{\partial C(x, y, z, t)}{\partial y}\right|_{x=L_{y}}=0,\left.\frac{\partial C(x, y, z, t)}{\partial z}\right|_{z=0}=0,\left.\frac{\partial C(x, y, z, t)}{\partial z}\right|_{x=L_{z}}=0 .
\end{gathered}
$$

Here $C(x, y, z, t)$ is the spatio-temporal distribution of concentration of dopant; $x, y$ and $z$ are current coordinates, $t$ is the current time; $T$ is the temperature of annealing; $D_{C}$ is the dopant diffusion coefficient. Value of dopant diffusion coefficient depends on properties of materials of heterostructure, speed of heating and cooling of the heterostructure (with account Arrhenius law). Dependences of dopant diffusion coefficient on parameters could be approximated by the following relation [26-28] 


$$
D_{C}=D_{L}(x, y, z, T)\left[1+\xi \frac{C^{\gamma}(x, y, z, t)}{P^{\gamma}(x, y, z, T)}\right]\left[1+\varsigma_{1} \frac{V(x, y, z, t)}{V^{*}}+\varsigma_{2} \frac{V^{2}(x, y, z, t)}{\left(V^{*}\right)^{2}}\right],
$$

where $D_{L}(x, y, z, T)$ is the spatial (due to presents several layers with different properties framework heterostructure) and temperature (due to Arrhenius law) dependences of dopant diffusion coefficient; $P(x, y, z, T)$ is the limit of solubility of dopant; parameter $\gamma$ depends on properties of materials and could be integer in the following interval $\gamma \in[1,3][26] ; V(x, y, z, t)$ is the spatio- temporal distribution of radiation vacancies; $V^{*}$ is the equilibrium distribution of vacancies. Concentrational dependence of dopant diffusion coefficient is describes in details in [26]. It should be noted, that diffusion type of doping did not leads to generation of radiation defects. In this situation $\zeta_{1}=$ $\zeta_{2}=0$. We determine spatio-temporal distributions of concentrations of point radiation defects as solutions of the following system of equations [27,28]

$$
\left\{\begin{array}{l}
\frac{\partial I(x, y, z, t)}{\partial t}=\frac{\partial}{\partial x}\left[D_{I}(x, y, z, T) \frac{\partial I(x, y, z, t)}{\partial x}\right]+\frac{\partial}{\partial y}\left[D_{I}(x, y, z, T) \frac{\partial I(x, y, z, t)}{\partial y}\right]+ \\
+\frac{\partial}{\partial z}\left[D_{I}(x, y, z, T) \frac{\partial I(x, y, z, t)}{\partial z}\right]-k_{I, V}(x, y, z, T) I(x, y, z, t) V(x, y, z, t)- \\
-k_{I, I}(x, y, z, T) I^{2}(x, y, z, t) \\
\frac{\partial V(x, y, z, t)}{\partial t}=\frac{\partial}{\partial x}\left[D_{V}(x, y, z, T) \frac{\partial V(x, y, z, t)}{\partial x}\right]+\frac{\partial}{\partial y}\left[D_{V}(x, y, z, T) \frac{\partial V(x, y, z, t)}{\partial y}\right]+ \\
+\frac{\partial}{\partial z}\left[D_{V}(x, y, z, T) \frac{\partial V(x, y, z, t)}{\partial z}\right]-k_{I, V}(x, y, z, T) I(x, y, z, t) V(x, y, z, t)- \\
-k_{V, V}(x, y, z, T) V^{2}(x, y, z, t)
\end{array}\right.
$$

with boundary and initial conditions

$$
\begin{gathered}
\left.\frac{\partial I(x, y, z, t)}{\partial x}\right|_{x=0}=0,\left.\frac{\partial I(x, y, z, t)}{\partial x}\right|_{x=L_{x}}=0,\left.\frac{\partial I(x, y, z, t)}{\partial y}\right|_{y=0}=0,\left.\frac{\partial I(x, y, z, t)}{\partial y}\right|_{y=L_{y}}=0, \\
\left.\frac{\partial I(x, y, z, t)}{\partial z}\right|_{z=0}=0,\left.\frac{\partial I(x, y, z, t)}{\partial z}\right|_{z=L_{z}}=0,\left.\frac{\partial V(x, y, z, t)}{\partial x}\right|_{x=0}=0,\left.\frac{\partial V(x, y, z, t)}{\partial x}\right|_{x=L_{x}}=0, \\
\left.\frac{\partial V(x, y, z, t)}{\partial y}\right|_{y=0}=0,\left.\frac{\partial V(x, y, z, t)}{\partial y}\right|_{y=L_{y}}=0,\left.\frac{\partial V(x, y, z, t)}{\partial z}\right|_{z=0}=0,\left.\frac{\partial V(x, y, z, t)}{\partial z}\right|_{z=L_{z}}=0, \\
I(x, y, z, 0)=f_{I}(x, y, z), V(x, y, z, 0)=f_{V}(x, y, z) .
\end{gathered}
$$

Here $\rho=I, V ; I(x, y, z, t)$ is the spatio-temporal distribution of concentration of radiation interstitials; $D_{\rho}(x, y, z, T)$ are the diffusion coefficients of vacancies and interstitials; terms $V^{2}(x, y, z, t)$ and $I^{2}(x, y, z, t)$ correspond to generation of divacancies and diinterstitials; $k_{I, V}(x, y, z, T), k_{I, I}(x, y, z, T)$ and $k_{V, V}(x, y, z, T)$ are parameters of recombination of point radiation defects and generation their complexes. Spatio-temporal distributions of concentrations of simplest complexes (divacancies $\Phi_{V}(x, y, z, t)$ and diinterstitials $\left.\Phi_{I}(x, y, z, t)\right)$ of radiation defects have been determined by solving the following system of equations $[27,28]$

$$
\frac{\partial \Phi_{I}(x, y, z, t)}{\partial t}=\frac{\partial}{\partial x}\left[D_{\Phi I}(x, y, z, T) \frac{\partial \Phi_{I}(x, y, z, t)}{\partial x}\right]+\frac{\partial}{\partial y}\left[D_{\Phi I}(x, y, z, T) \frac{\partial \Phi_{I}(x, y, z, t)}{\partial y}\right]+
$$




$$
\begin{aligned}
& +\frac{\partial}{\partial z}\left[D_{\Phi I}(x, y, z, T) \frac{\partial \Phi_{I}(x, y, z, t)}{\partial z}\right]+k_{I, I}(x, y, z, T) I^{2}(x, y, z, t)-k_{I}(x, y, z, T) I(x, y, z, t) \\
& \frac{\partial \Phi_{V}(x, y, z, t)}{\partial t}=\frac{\partial}{\partial x}\left[D_{\Phi V}(x, y, z, T) \frac{\partial \Phi_{V}(x, y, z, t)}{\partial x}\right]+\frac{\partial}{\partial y}\left[D_{\Phi V}(x, y, z, T) \frac{\partial \Phi_{V}(x, y, z, t)}{\partial y}\right]+ \\
& +\frac{\partial}{\partial z}\left[D_{\Phi V}(x, y, z, T) \frac{\partial \Phi_{V}(x, y, z, t)}{\partial z}\right]+k_{V, V}(x, y, z, T) V^{2}(x, y, z, t)-k_{V}(x, y, z, T) V(x, y, z, t)
\end{aligned}
$$

with boundary and initial conditions

$$
\begin{gathered}
\left.\frac{\partial \Phi_{\rho}(x, y, z, t)}{\partial x}\right|_{x=0}=0,\left.\frac{\partial \Phi_{\rho}(x, y, z, t)}{\partial x}\right|_{x=L_{x}}=0,\left.\frac{\partial \Phi_{\rho}(x, y, z, t)}{\partial y}\right|_{y=0}=0, \\
\left.\frac{\partial \Phi_{\rho}(x, y, z, t)}{\partial y}\right|_{y=L_{y}}=0,\left.\frac{\partial \Phi_{\rho}(x, y, z, t)}{\partial z}\right|_{z=0}=0,\left.\frac{\partial \Phi_{\rho}(x, y, z, t)}{\partial z}\right|_{z=L_{z}}=0, \\
\Phi_{I}(x, y, z, 0)=f_{\Phi I}(x, y, z), \Phi_{V}(x, y, z, 0)=f_{\Phi V}(x, y, z) .
\end{gathered}
$$

Here $D_{\Phi \rho}(x, y, z, T)$ are the diffusion coefficients of complexes of radiation defects; $k_{I}(x, y, z, T)$ and $k_{V}(x, y, z, T)$ are the parameters of decay of complexes of radiation defects.

We determine spatio-temporal distribution of temperature by solving of the second law of Fourier [29]

$$
\begin{aligned}
c(T) \frac{\partial T(x, y, z, t)}{\partial t}= & \frac{\partial}{\partial x}\left[\lambda(x, y, z, T) \frac{\partial T(x, y, z, t)}{\partial x}\right]+\frac{\partial}{\partial y}\left[\lambda(x, y, z, T) \frac{\partial T(x, y, z, t)}{\partial y}\right]+ \\
& +\frac{\partial}{\partial z}\left[\lambda(x, y, z, T) \frac{\partial T(x, y, z, t)}{\partial z}\right]+p(x, y, z, t),
\end{aligned}
$$

with boundary and initial conditions

$$
\begin{gathered}
\left.\frac{\partial T(x, y, z, t)}{\partial x}\right|_{x=0}=0,\left.\frac{\partial T(x, y, z, t)}{\partial x}\right|_{x=L_{x}}=0,\left.\frac{\partial T(x, y, z, t)}{\partial y}\right|_{y=0}=0,\left.\frac{\partial T(x, y, z, t)}{\partial y}\right|_{x=L_{y}}=0, \\
\left.\frac{\partial T(x, y, z, t)}{\partial z}\right|_{z=0}=0,\left.\frac{\partial T(x, y, z, t)}{\partial z}\right|_{x=L_{z}}=0, T(x, y, z, 0)=f_{T}(x, y, z),
\end{gathered}
$$

where $T(x, y, z, t)$ is the spatio-temporal distribution of temperature of annealing; $c(T)=c_{a s s}[1-\eta$ $\left.\exp \left(-T(x, y, z, t) / T_{d}\right)\right]$ is the heat capacitance (in the most interest case, when current temperature is approximately equal or larger, than Debye temperature $T_{d}$, we have possibility to consider the following limiting case $c(T) \approx c_{a s s}$ ) [29]; $\lambda$ is the heat conduction coefficient, which depends on properties of materials and temperature (dependence on temperature of the heat conduction coefficient could be approximated by the function $\lambda(x, y, z, T)=\lambda_{a s s}(x, y, z)\left[1+\mu\left(T_{d} / T(x, y, z, t)\right)^{\varphi}\right]$ in the most interesting interval of temperature [29]); $p(x, y, z, t)$ is the volumetric density of power of heating; $\alpha(x, y, z, T)=\lambda(x, y, z, T) / c(T)$ is the thermal diffusivity.

First of all we calculate spatio-temporal distribution of temperature. To make the calculation we used recently considered approach $[17,20,30]$. Framework the approach we transform approximation of thermal diffusivity to the following form: $\alpha_{a s s}(x, y, z)=\lambda_{\text {ass }}(x, y, z) / c_{\text {ass }}=\alpha_{0 a s s}\left[1+\varepsilon_{T} g_{T}(x, y, z)\right]$. Farther we determine solution of the Eq.(8) as the following power series $[17,20,30]$ 


$$
T(x, y, z, t)=\sum_{i=0}^{\infty} \varepsilon_{T}^{i} \sum_{j=0}^{\infty} \mu^{j} T_{i j}(x, y, z, t)
$$

Substitution of the series into Eq.(8) gives us possibility to obtain system of equations for initialorder approximation of temperature $T_{00}(x, y, z, t)$ and corrections for them $T_{i j}(x, y, z, t)(i \geq 1, j \geq 1)$. The equations are presented in the Appendix. Substitution of the series (10) into boundary and initial conditions (9) gives us possibility to obtain boundary and initial conditions for all functions $T_{i j}(x, y, z, t)(i \geq 0, j \geq 0)$. The solutions are presented in the Appendix. Solutions of the equations for the functions $T_{i j}(x, y, z, t)(i \geq 0, j \geq 0)$ have been calculated by standard approaches [31,32] framework the second-order approximation on parameters $\varepsilon$ and $\mu$. The solutions are presented in the Appendix. To make qualitative analysis and to obtain some quantitative results the second-order approximation is usually enough good approximation (see, for example, [17,20,30]). Analytical results give us possibility to obtain and demonstrably. All analytical results have been checked by comparison with numerical one.

To determine spatio-temporal distributions of concentrations of point defects we used recently introduces approach $[17,20,30]$. Framework the approach we transform approximation of their diffusion coefficients to the following form $D_{\rho}(x, y, z, T)=D_{0 \rho}\left[1+\varepsilon_{\rho} g_{\rho}(x, y, z, T)\right]$, where $D_{0 \rho}$ are the average values of the diffusion coefficients, $0 \leq \varepsilon_{\rho}<1,\left|g_{\rho}(x, y, z, T)\right| \leq 1, \rho=I, V$. Analogous transformation of approximations of parameters of recombination of point defects and generation their complexes has been used: $k_{I, V}(x, y, z, T)=k_{0 I, V}\left[1+\varepsilon_{I, V} g_{I, V}(x, y, z, T)\right], k_{I, I}(x, y, z, T)=k_{0 I, I}\left[1+\varepsilon_{I, I} g_{I, I}(x, y, z, T)\right]$ and $k_{V, V}(x, y, z, T)=k_{0 V, V}\left[1+\varepsilon_{V, V} g_{V, V}(x, y, z, T)\right]$, where $k_{0 \rho 1, \rho 2}$ are the appropriate average values, $0 \leq \varepsilon_{I, V}<1,0 \leq \varepsilon_{I, I}<1,0 \leq \varepsilon_{V, V}<1,\left|g_{I, V}(x, y, z, T)\right| \leq 1,\left|g_{I, I}(x, y, z, T)\right| \leq 1,\left|g_{V, V}(x, y, z, T)\right| \leq 1$. Let us introduce following dimensionless variables: $\tilde{I}(x, y, z, t)=I(x, y, z, t) / I^{*}, \tilde{V}(x, y, z, t)=V(x, y, z, t) / V^{*}, \chi=$ $x / L_{x}, \eta=y / L_{y}, \phi=z / L_{z}, \vartheta=\sqrt{D_{0 I} D_{0 V}} t / L^{2}, \omega=L^{2} k_{0 I, V} / \sqrt{D_{0 I} D_{0 V}}, \Omega_{\rho}=L^{2} k_{0 \rho, \rho} / \sqrt{D_{0 I} D_{0 V}}$. The introduction leads to following transformation of Eqs.(4) and conditions (5)

$$
\begin{gathered}
\frac{\partial \tilde{I}(\chi, \eta, \phi, \vartheta)}{\partial \vartheta}=\frac{D_{0 I}}{\sqrt{D_{0 I} D_{0 V}}} \frac{\partial}{\partial \chi}\left\{\left[1+\varepsilon_{I} g_{I}(\chi, \eta, \phi, T)\right] \frac{\partial \tilde{I}(\chi, \eta, \phi, \vartheta)}{\partial \chi}\right\}+\frac{D_{0 I}}{\sqrt{D_{0 I} D_{0 V}}} \times \\
\times \frac{\partial}{\partial \eta}\left\{\left[1+\varepsilon_{I} g_{I}(\chi, \eta, \phi, T)\right] \frac{\partial \tilde{I}(\chi, \eta, \phi, \vartheta)}{\partial \eta}\right\}+\frac{D_{0 I}}{\sqrt{D_{0 I} D_{0 V}}} \frac{\partial}{\partial \phi}\left\{\left[1+\varepsilon_{I} g_{I}(\chi, \eta, \phi, T)\right] \frac{\partial \tilde{I}(\chi, \eta, \phi, \vartheta)}{\partial \phi}\right\}- \\
-\omega\left[1+\varepsilon_{I, V} g_{I, V}(\chi, \eta, \phi, T)\right] \tilde{I}(\chi, \eta, \phi, \vartheta) \tilde{V}(\chi, \eta, \phi, \vartheta)-\Omega_{I} \tilde{I}^{2}(\chi, \eta, \phi, \vartheta)\left[1+\varepsilon_{I, I} g_{I, I}(\chi, \eta, \phi, T)\right](11) \\
\frac{\partial \tilde{V}(\chi, \eta, \phi, \vartheta)}{\partial \vartheta}=\frac{D_{0 V}}{\sqrt{D_{0 I} D_{0 V}}} \frac{\partial}{\partial \chi}\left\{\left[1+\varepsilon_{V} g_{V}(\chi, \eta, \phi, T)\right] \frac{\partial \tilde{V}(\chi, \eta, \phi, \vartheta)}{\partial \chi}\right\}+\frac{D_{0 V}}{\sqrt{D_{0 I} D_{0 V}} \times} \\
\times \frac{\partial}{\partial \eta}\left\{\left[1+\varepsilon_{V} g_{V}(\chi, \eta, \phi, T)\right] \frac{\partial \tilde{V}(\chi, \eta, \phi, \vartheta)}{\partial \eta}\right\}+\frac{\partial}{\partial \phi}\left\{\left[1+\varepsilon_{V} g_{V}(\chi, \eta, \phi, T)\right] \frac{\partial \tilde{V}(\chi, \eta, \phi, \vartheta)}{\partial \phi}\right\} \frac{D_{0 V}}{\sqrt{D_{0 I} D_{0 V}}}- \\
-\omega\left[1+\varepsilon_{I, V} g_{I, V}(\chi, \eta, \phi, T)\right] \tilde{I}(\chi, \eta, \phi, \vartheta) \tilde{V}(\chi, \eta, \phi, \vartheta)-\Omega_{V} \tilde{V}{ }^{2}(\chi, \eta, \phi, \vartheta)\left[1+\varepsilon_{V, V} g_{V, V}(\chi, \eta, \phi, T)\right] \\
\left.\frac{\partial \tilde{\rho}(\chi, \eta, \phi, \vartheta)}{\partial \chi}\right|_{\chi=0}=0,\left.\frac{\partial \tilde{\rho}(\chi, \eta, \phi, \vartheta)}{\partial \chi}\right|_{\chi=1}=0,\left.\frac{\partial \tilde{\rho}(\chi, \eta, \phi, \vartheta)}{\partial \eta}\right|_{\eta=0}=0,\left.\frac{\partial \tilde{\rho}(\chi, \eta, \phi, \vartheta)}{\partial \eta}\right|_{\eta=1}=0, \\
\left.\frac{\partial \tilde{\rho}(\chi, \eta, \phi, \vartheta)}{\partial \phi}\right|_{\phi=0}=0,\left.\frac{\partial \tilde{\rho}(\chi, \eta, \phi, \vartheta)}{\partial \phi}\right|_{\phi=1}=0, \tilde{\rho}(\chi, \eta, \phi, \vartheta)=\frac{f_{\rho}(\chi, \eta, \phi, \vartheta)}{\rho{ }^{*}} .
\end{gathered}
$$


We solved the Eqs.(11) with conditions (12) by using the approach, considered in $[17,20,30]$. Framework the approach we determine solutions of the equations as the following power series

$$
\tilde{\rho}(\chi, \eta, \phi, \vartheta)=\sum_{i=0}^{\infty} \varepsilon_{\rho}^{i} \sum_{j=0}^{\infty} \omega^{j} \sum_{k=0}^{\infty} \Omega_{\rho}^{k} \tilde{\rho}_{i j k}(\chi, \eta, \phi, \vartheta) .
$$

Substitution of the series (13) into Eqs.(11) and conditions (12) gives us possibility to obtain equations for initial-order approximations of concentrations of point radiation defects $\tilde{\rho}_{000}(\chi, \eta, \phi, \vartheta)$ and corrections for them $\tilde{\rho}_{i j k}(\chi, \eta, \phi, \vartheta), i \geq 1, j \geq 1, k \geq 1$. The equations and conditions for them are presented in the Appendix. The equations with account the conditions have been solved by standard approaches (see, for example, Fourier approach [31,32]). The solutions are presented in the Appendix.

Farther we calculate spathio-temporal distributions of concentrations of complexes of radiation defects. To determine these distributions we transform approximations of diffusion coefficients to the following form: $D_{\Phi \rho}(x, y, z, T)=D_{0 \Phi_{\rho}}\left[1+\varepsilon_{\Phi \rho} g_{\Phi \rho}(x, y, z, T)\right]$, where $D_{0 \Phi_{\rho}}$ are the average values of the coefficients. In this situation the Eqs. (6) have been transform to the following form

$$
\begin{gathered}
\frac{\partial \Phi_{I}(x, y, z, t)}{\partial t}=D_{0 \Phi_{I}} \frac{\partial}{\partial x}\left\{\left[1+\varepsilon_{\Phi_{I}} g_{\Phi_{I}}(x, y, z, T)\right] \frac{\partial \Phi_{I}(x, y, z, t)}{\partial x}\right\}+D_{0 \Phi_{I}} \times \\
\times \frac{\partial}{\partial y}\left\{\left[1+\varepsilon_{\Phi_{I}} g_{\Phi_{I}}(x, y, z, T)\right] \frac{\partial \Phi_{I}(x, y, z, t)}{\partial y}\right\}+\frac{\partial}{\partial z}\left\{\left[1+\varepsilon_{\Phi_{I}} g_{\Phi_{I}}(x, y, z, T)\right] \frac{\partial \Phi_{I}(x, y, z, t)}{\partial z}\right\} \times \\
\times D_{0 \Phi_{I}}+k_{I, I}(x, y, z, T) I^{2}(x, y, z, t)-k_{I}(x, y, z, T) I(x, y, z, t) \\
\frac{\partial \Phi_{V}(x, y, z, t)}{\partial t}=D_{0 \Phi_{V}} \frac{\partial}{\partial x}\left\{\left[1+\varepsilon_{0 \Phi_{V}} g_{0 \Phi_{V}}(x, y, z, T)\right] \frac{\partial \Phi_{V}(x, y, z, t)}{\partial x}\right\}+D_{0 \Phi_{V}} \times \\
\times \frac{\partial}{\partial y}\left\{\left[1+\varepsilon_{\Phi_{V}} g_{\Phi_{V}}(x, y, z, T)\right] \frac{\partial \Phi_{V}(x, y, z, t)}{\partial y}\right\}+\frac{\partial}{\partial z}\left\{\left[1+\varepsilon_{\Phi_{V}} g_{\Phi_{V}}(x, y, z, T)\right] \frac{\partial \Phi_{V}(x, y, z, t)}{\partial z}\right\} \times \\
\times D_{0 \Phi_{V}}+k_{V, V}(x, y, z, T) V^{2}(x, y, z, t)-k_{V}(x, y, z, T) V(x, y, z, t) .
\end{gathered}
$$

Let us determine solutions of the above equations in the following form

$$
\Phi_{\rho}(x, y, z, t)=\sum_{i=0}^{\infty} \varepsilon_{\Phi \rho}^{i} \Phi_{\rho i}(x, y, z, t) .
$$

Substitution of the series (14) into Eqs.(6) and appropriate boundary and initial conditions gives us possibility to obtain equations for all functions $\Phi_{\rho i}(x, y, z, t), i \geq 1$, boundary and initial conditions for them. The equations and conditions are presented in the Appendix. The equation have been solved by standard approaches [31,32]. The obtained solutions are presented in the Appendix.

To calculate spatio-temporal distribution of concentration of dopant we transform approximation of dopant diffusion coefficient in the following form: $D_{L}(x, y, z, T)=D_{0 L}\left[1+\varepsilon_{L} g_{L}(x, y, z, T)\right]$, where $D_{0 L}$ is the average value of dopant diffusion coefficient, $0 \leq \varepsilon_{L}<1,\left|g_{L}(x, y, z, T)\right| \leq 1$. Farther we determine solution of Eq.(1) as the following power series

$$
C(x, y, z, t)=\sum_{i=0}^{\infty} \varepsilon_{L}^{i} \sum_{j=1}^{\infty} \xi^{j} C_{i j}(x, y, z, t)
$$


Substitution of the series in the Eq.(1) and condition (2) gives us possibility to obtain equation for initial-order approximation of dopant concentration $C_{00}(x, y, z, t)$, equations for corrections for them $C_{i j}(x, y, z, t)(i \geq 1, j \geq 1)$, boundary and initial conditions for all functions $C_{i j}(x, y, z, t)(i \geq 0, j \geq 0)$. The equations and conditions are presented in the Appendix. The equations have been solved by standard approaches (see, for example, [31,32]). The solutions are presented in the Appendix.

Analysis of spatiotemporal distributions of concentrations of dopant and radiation defects and temperature have been done analytically by using the second-order approximations on all considered parameters. The approximations are usually enough good approximations to make qualitative analysis and to obtain some quantitative results (see, for example, [17,20,30]). Analytical results give us possibility to obtain and demonstrably. All analytical results have been checked by comparison with numerical one.

\section{Discussion}

In this section we analyzed dynamic of redistribution of dopant and radiation defects in the considered heterostructure during their annealing by using calculated in the previous section relations. Typical distributions of concentrations of dopant in heterostructures are presented on Figs. 2 and 3 for diffusion and ion types of doping, respectively. These distributions have been calculated for the case, when value of dopant diffusion coefficient in doped area is larger, than in nearest areas. The figures show, that inhomogeneity of heterostructure gives us possibility to increase sharpness of $p-n$-junctions. At the same time one can find increasing of homogeneity of dopant distribution in doped part of epitaxial layer. Increasing of sharpness of $p$ - $n$-junctions gives us possibility to decrease their switching time. Increasing of homogeneity of dopant distribution in doped are gives us possibility to decrease local overheat of doped material during functioning of $p$ - $n$-junction or decreasing of dimensions of the $p$ - $n$-junction for fixed maximal value of local overheat. On the other hand inhomogeneity of doping of base of transistor leads to generation an electric field in the base. The electric field gives us possibility to accelerate transport of charge carriers. The acceleration gives us possibility to increase performance of the transistor.

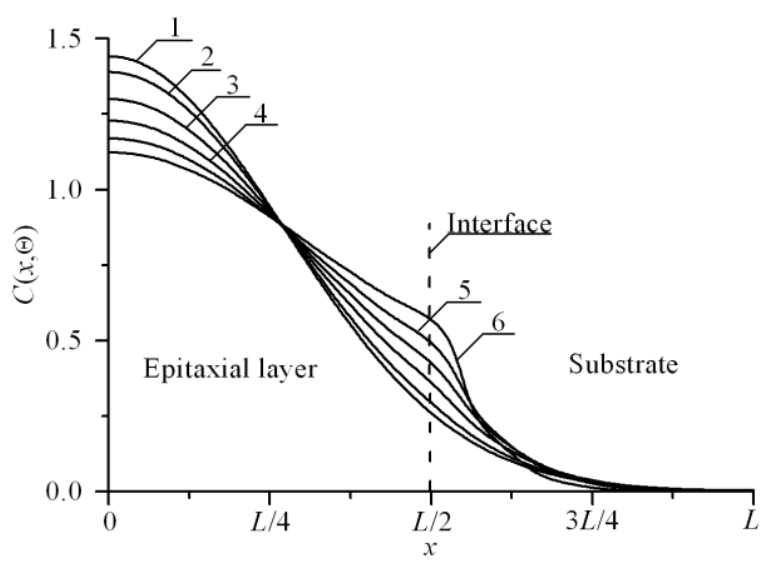

Fig.2. Distributions of concentration of infused dopant in heterostructure from Fig. 1 in direction, which is perpendicular to interface between epitaxial layer substrate. Increasing of number of curve corresponds to increasing of difference between values of dopant diffusion coefficient in layers of heterostructure under condition, when value of dopant diffusion coefficient in epitaxial layer is larger, than value of dopant diffusion coefficient in substrate 


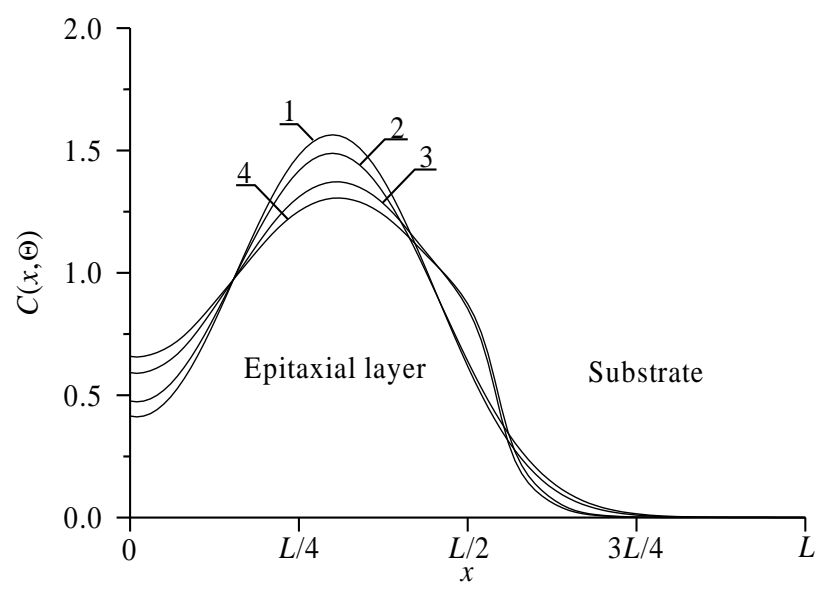

Fig.3. Distributions of concentration of implanted dopant in heterostructure from Fig. 1 in direction, which is perpendicular to interface between epitaxial layer substrate. Curves 1 and 3 corresponds to annealing time $\Theta=0.0048\left(L_{x}{ }^{2}+L_{y}{ }^{2}+L_{z}{ }^{2}\right) / D_{0}$. Curves 2 and 4 corresponds to annealing time $\Theta=0.0057\left(L_{x}{ }^{2}+L_{y}{ }^{2}+L_{z}{ }^{2}\right) / D_{0}$. Curves 1 and 2 corresponds to homogenous sample. Curves 3 and 4 corresponds to heterostructure under condition, when value of dopant diffusion coefficient in epitaxial layer is larger, than value of dopant diffusion coefficient in substrate

To help in manufacturing of the drift transistors it could be used microwave annealing. Using this type of annealing give us possibility to generate inhomogenous distribution of temperature. The inhomogeneity gives us possibility to form more inhomogenous distribution of concentration of dopant due to Arrhenius law [33]. Using microwave annealing leads to necessity to choose frequency of electro-magnetic irradiation so, that thickness of scin-layer should be larger, than thickness of external epitaxial layer, and smaller, than sum of thicknesses of external and average epitaxial layers.

It should be noted, that increasing of annealing time leads to increasing of homogeneity of distribution of concentration of dopant. In this situation the distribution became too large. If annealing time is too small, dopant has not time to achieve nearest interface between materials of heterostructure. In this situation distribution of concentration of dopant has not any changing. In this situation it is attracted an interest optimization of annealing. We consider the optimization of annealing framework recently introduced criterion [17-24,33]. Framework the criterion we approximate real distributions of concentrations of dopants by step-wise function $\psi(x, y, z)$. Farther we minimize the following mean-squared error to optimize annealing time

$$
U=\frac{1}{L_{x} L_{y} L_{z}} \int_{0}^{L_{x}} \int_{0}^{L_{y}} \int_{0}^{L_{z}}[C(x, y, z, \Theta)-\psi(x, y, z)] d z d y d x
$$




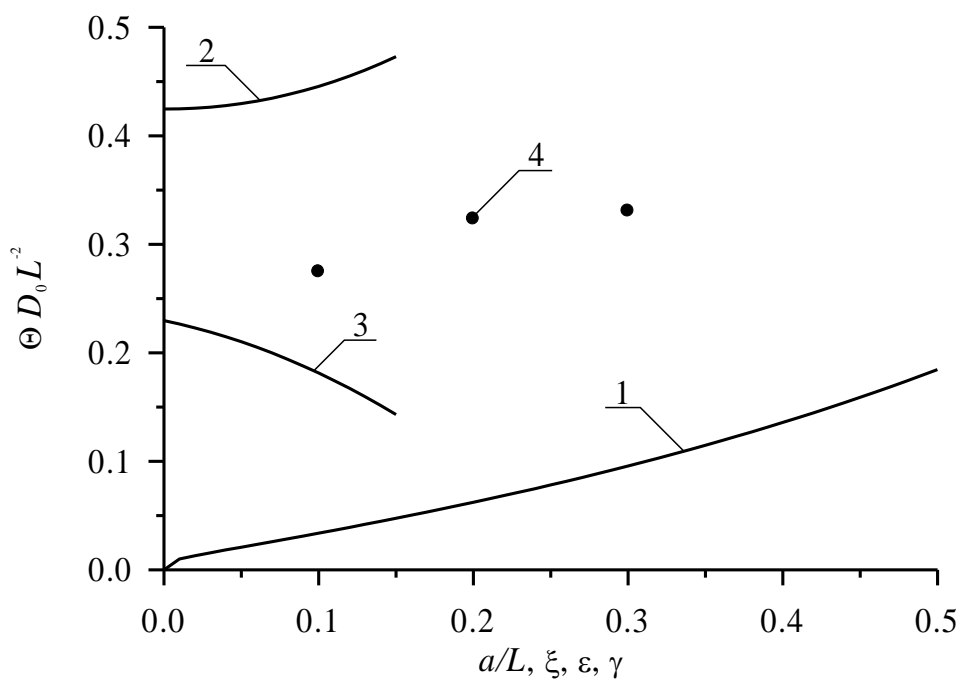

Fig.4. Dependences of dimensionless optimal annealing time for doping by diffusion, which have been obtained by minimization of mean-squared error, on several parameters. Curve 1 is the dependence of dimensionless optimal annealing time on the relation $a / L$ and $\xi=\gamma=0$ for equal to each other values of dopant diffusion coefficient in all parts of heterostructure. Curve 2 is the dependence of dimensionless optimal annealing time on value of parameter $\varepsilon$ for $a / L=1 / 2$ and $\xi=\gamma=0$. Curve 3 is the dependence of dimensionless optimal annealing time on value of parameter $\xi$ for $a / L=1 / 2$ and $\varepsilon=\gamma=0$. Curve 4 is the dependence of dimensionless optimal annealing time on value of parameter $\gamma$ for $a / L=1 / 2$ and $\varepsilon=\xi=0$

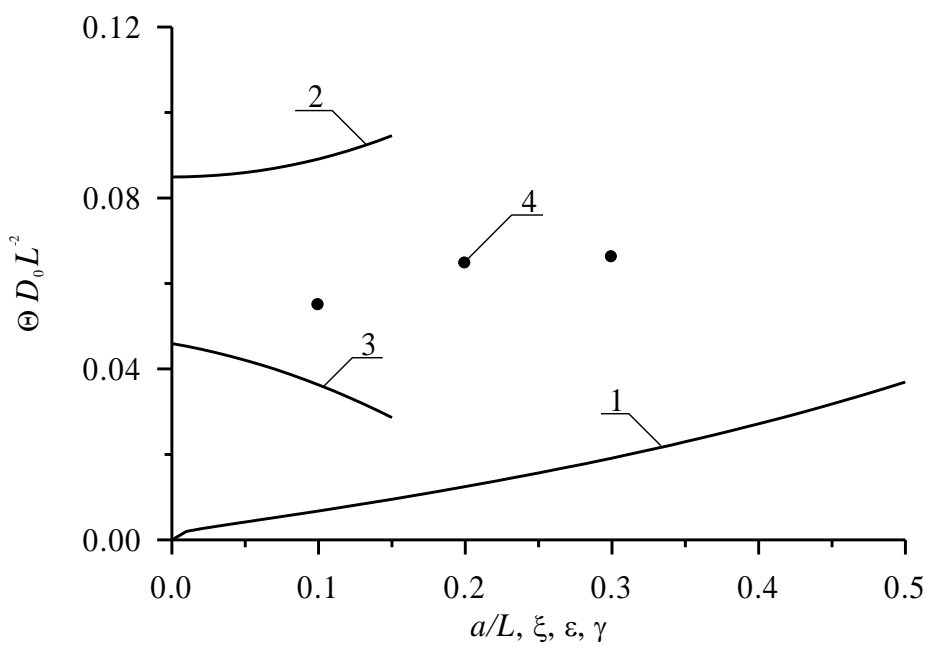

Fig.5. Dependences of dimensionless optimal annealing time for doping by ion implantation, which have been obtained by minimization of mean-squared error, on several parameters. Curve 1 is the dependence of dimensionless optimal annealing time on the relation $a / L$ and $\xi=\gamma=0$ for equal to each other values of dopant diffusion coefficient in all parts of heterostructure. Curve 2 is the dependence of dimensionless optimal annealing time on value of parameter $\varepsilon$ for $a / L=1 / 2$ and $\xi=\gamma=0$. Curve 3 is the dependence of dimensionless optimal annealing time on value of parameter $\xi$ for $a / L=1 / 2$ and $\varepsilon=\gamma=0$. Curve 4 is the dependence of dimensionless optimal annealing time on value of parameter $\gamma$ for $a / L=1 / 2$ and $\varepsilon=\xi=0$

Dependences of optimal values of annealing time are presented on Figs. 4 and 5. Optimal values of annealing time of implanted dopant is smaller, than optimal values of annealing time of infused dopant. The difference existing due to necessity of annealing of radiation defects. During annealing radiation defects one can find spreading of distribution of concentration of dopant. In the ideal 
case during annealing of radiation defects should achieves interface between materials of heterostructure. If dopant has no time to achieve the interface, additional annealing of dopant attracted an interest. The Fig. 5 shows exactly dependences of time of additional annealing.

\section{Conclusions}

In this paper we consider an approach to manufacture more compact elements of emitter-coupled logic. The approach based on manufacturing a heterostructure with required configuration, doping of required areas of the heterostructure by diffusion or ion implantation and optimization of annealing of dopant and/or radiation defects.

\section{ACKNOWLEDGEMENTS}

This work is supported by the contract 11.G34.31.0066 of the Russian Federation Government, grant of Scientific School of Russia, the agreement of August 27, 2013 № 02.B.49.21.0003 between The Ministry of education and science of the Russian Federation and Lobachevsky State University of Nizhni Novgorod and educational fellowship for scientific research of Nizhny Novgorod State University of Architecture and Civil Engineering.

\section{REFERENCES}

[1] V.I. Lachin, N.S. Savelov. Electronics. Rostov-on-Don: Phoenix, 2001.

[2] A. Polishscuk. Analog integrated circuits Anadigm: the whole range of analog electronics on a single chip. The first acquaintance. Modern Electronics. Issue 12. P. 8-11 (2004).

[3] G. Volovich. Modern chips UM3Ch class D manufactured by firm MPS. Modern Electronics. Issue 2. P. 10-17 (2006).

[4] A. Kerentsev, V. Lanin, Constructive-technological features of MOSFET-transistors. Power Electronics. Issue 1. P. 34 (2008).

[5] A.O. Ageev, A.E. Belyaev, N.S. Boltovets, V.N. Ivanov, R.V. Konakova, Ya.Ya. Kudrik, P.M. Litvin, V.V. Milenin, A.V. Sachenko. Au-TiBx-n-6H-SiC Schottky barrier diodes: the features of current flow in rectifying and nonrectifying contacts. Semiconductors. Vol. 43 (7). P. 897-903 (2009).

[6] Jung-Hui Tsai, Shao-Yen Chiu, Wen-Shiung Lour, Der-Feng Guo. High-performance InGaP/GaAs pnp $\delta$-doped heterojunction bipolar transistor. Semiconductors. Vol. 43 (7). P. 971-974 (2009).

[7] O.V. Alexandrov, A.O. Zakhar'in, N.A. Sobolev, E.I. Shek, M.M. Makoviychuk, E.O. Parshin. Formation of donor centers after annealing of dysprosium and holmium implanted silicon. Semiconductors. Vol. 32 (9). P. 1029-1032 (1998).

[8] I.B. Ermolovich, V.V. Milenin, R.A. Red'ko, S.M. Red'ko. Features of recombination processes in CdTe films, preparing at different temperature conditions and further annealing. Semiconductors. Vol. 43 (8). P. 1016-1020 (2009).

[9] P. Sinsermsuksakul, K. Hartman, S.B. Kim, J. Heo, L. Sun, H.H. Park, R. Chakraborty, T. Buonassisi, R.G. Gordon. Enhancing the efficiency of SnS solar cells via band-offset engineering with a zinc oxysulfide buffer layer. Appl. Phys. Lett. Vol. 102 (5). P. $053901-053905$ (2013).

[10] J.G. Reynolds, C.L. Reynolds, Jr.A. Mohanta, J.F. Muth, J.E. Rowe, H.O. Everitt, D.E. Aspnes. Shallow acceptor complexes in p-type ZnO. Appl. Phys. Lett. Vol. 102 (15). P. 152114-152118 (2013).

[11] K.K. Ong, K.L. Pey, P.S. Lee, A.T.S. Wee, X.C. Wang, Y.F. Chong. Dopant distribution in the recrys-tallization transient at the maximum melt depth induced by laser annealing. Appl. Phys. Lett. 89 (17). P. 172111-172114 (2006).

[12] H.T. Wang, L.S. Tan, E. F. Chor. Pulsed laser annealing of Be-implanted GaN. J. Appl. Phys. 98 (9). P. 094901-094905 (2006).

[13] Yu.V. Bykov, A.G. Yeremeev, N.A. Zharova, I.V. Plotnikov, K.I. Rybakov, M.N. Drozdov, Yu.N. Drozdov, V.D. Skupov. Diffusion processes in semiconductor structures during microwave annealing. Radiophysics and Quantum Electronics. Vol. 43 (3). P. 836-843 (2003).

[14] I.P. Stepanenko. Basis of Microelectronics (Soviet Radio, Moscow, 1980).

[15] A.G. Alexenko, I.I. Shagurin. Microcircuitry. Moscow: Radio and communication, 1990. 
[16] N.A. Avaev, Yu.E. Naumov, V.T. Frolkin. Basis of microelectronics (Radio and communication, Moscow, 1991).

[17] E.L. Pankratov. Dopant diffusion dynamics and optimal diffusion time as influenced by diffusioncoefficient nonuniformity. Russian Microelectronics. 2007. V.36 (1). P. 33-39.

[18] E.L. Pankratov. Redistribution of dopant during annealing of radiative defects in a multilayer structure by laser scans for production an implanted-junction rectifiers. Int. J. Nanoscience. Vol. 7 (4-5). P. 187-197 (2008).

[19] E.L. Pankratov. Decreasing of depth of implanted-junction rectifier in semiconductor heterostructure by optimized laser annealing. J. Comp. Theor. Nanoscience. Vol. 7 (1). P. 289-295 (2010).

[20] E.L. Pankratov, E.A. Bulaeva. Doping of materials during manufacture p-n-junctions and bipolar transistors. Analytical approaches to model technological approaches and ways of optimization of distributions of dopants. Reviews in Theoretical Science. Vol. 1 (1). P. 58-82 (2013).

[21] E.L. Pankratov, E.A. Bulaeva. Application of native inhomogeneities to increase compactness of vertical field-effect transistors. J. Comp. Theor. Nanoscience. Vol. 10 (4). P. 888-893 (2013).

[22] E.L. Pankratov, E.A. Bulaeva. Optimization of doping of heterostructure during manufacturing of p-in-diodes. Nanoscience and Nanoengineering. Vol. 1 (1). P. 7-14 (2013).

[23] E.L. Pankratov, E.A. Bulaeva. An approach to decrease dimensions of field-effect transistors. Universal Journal of Materials Science. Vol. 1 (1). P.6-11 (2013).

[24] E.L. Pankratov, E.A. Bulaeva. An approach to manufacture a heterobipolar transistors in thin film structures. On the method of optimization. Int. J. Micro-Nano Scale Transp. Vol. 4 (1-2). P. 17-31 (2013).

[25] V.V. Kozlivsky. Modification of semiconductors by proton beams (Nauka, Sant-Peterburg, 2003, in Russian).

[26] Z.Yu. Gotra, Technology of microelectronic devices (Radio and communication, Moscow, 1991).

[27] V.L. Vinetskiy, G.A. Kholodar', Radiative physics of semiconductors. ("Naukova Dumka", Kiev, 1979, in Russian).

[28] P.M. Fahey, P.B. Griffin, J.D. Plummer. Point defects and dopant diffusion in silicon. Rev. Mod. Phys. 1989. Vol. 61. № 2. P. 289-388.

[29] K.V. Shalimova. Fisics of semiconductors (Energoatomizdat, Moscow, 1985, in Russian).

[30] E.L. Pankratov, E.A. Bulaeva. Decreasing of quantity of radiation defects in an implanted-junction rectifiers by using overlayers. Int. J. Micro-Nano Scale Transp. Vol. 3 (3). P. 119-130 (2012).

[31] A.N. Tikhonov, A.A. Samarskii. The mathematical physics equations (Moscow, Nauka 1972) (in Rus-sian).

[32] H.S. Carslaw, J.C. Jaeger. Conduction of heat in solids (Oxford University Press, 1964).

[33] E.L. Pankratov. Redistribution of dopant during microwave annealing of a multilayer structure for production p-n-junction. J. Appl. Phys. Vol. 103 (6). P. 064320-064330 (2008).

\section{Appendix}

Equations for initial-order approximation of temperature $T_{00}(x, y, z, t)$, corrections for the approximation $T_{i j}(x, y, z, t)(i \geq 0, j \geq 0)$, boundary and initial conditions for them have been obtain by substitution of the series (10) into Eqs.(8) and conditions (9) and equating of coefficients at equal powers of parameters, which have been used in the series (10). The equations and conditions could be written as

$$
\begin{aligned}
& \frac{\partial T_{00}(x, y, z, t)}{\partial t}=\alpha_{0 a s s}\left[\frac{\partial^{2} T_{00}(x, y, z, t)}{\partial x^{2}}+\frac{\partial^{2} T_{00}(x, y, z, t)}{\partial y^{2}}+\frac{\partial^{2} T_{00}(x, y, z, t)}{\partial z^{2}}\right]+\frac{p(x, y, z, t)}{v_{a s s}} \\
& \frac{\partial T_{i 0}(x, y, z, t)}{\partial t}=\alpha_{0 a s s}\left[\frac{\partial^{2} T_{i 0}(x, y, z, t)}{\partial x^{2}}+\frac{\partial^{2} T_{i 0}(x, y, z, t)}{\partial y^{2}}+\frac{\partial^{2} T_{i 0}(x, y, z, t)}{\partial z^{2}}\right]+ \\
& +\alpha_{0 a s s}\left\{g_{T}(z) \frac{\partial^{2} T_{i-10}(x, y, z, t)}{\partial x^{2}}+g_{T}(x) \frac{\partial^{2} T_{i-10}(x, y, z, t)}{\partial y^{2}}+\frac{\partial}{\partial z}\left[g_{T}(z) \frac{\partial T_{i-10}(x, y, z, t)}{\partial z}\right]\right\}, i \geq 1
\end{aligned}
$$


International Journal of Recent advances in Physics (IJRAP) Vol.4, No.2, May 2015

$$
\begin{aligned}
& \frac{\partial T_{01}(x, y, z, t)}{\partial t}=\alpha_{0 a s s}\left[\frac{\partial^{2} T_{01}(x, y, z, t)}{\partial x^{2}}+\frac{\partial^{2} T_{01}(x, y, z, t)}{\partial y^{2}}+\frac{\partial^{2} T_{01}(x, y, z, t)}{\partial z^{2}}\right]+\frac{\alpha_{0 a s s} T_{d}^{\varphi}}{T_{00}^{\varphi}(x, y, z, t)} \times \\
& \times\left[\frac{\partial^{2} T_{00}(x, y, z, t)}{\partial x^{2}}+\frac{\partial^{2} T_{00}(x, y, z, t)}{\partial y^{2}}+\frac{\partial^{2} T_{00}(x, y, z, t)}{\partial z^{2}}\right]-\frac{\alpha_{0 a s s} T_{d}^{\varphi}}{T_{00}^{\varphi}(x, y, z, t)}\left\{\left[\frac{\partial T_{00}(x, y, z, t)}{\partial x}\right]^{2}+\right. \\
& \left.+\left[\frac{\partial T_{00}(x, y, z, t)}{\partial y}\right]^{2}+\left[\frac{\partial T_{00}(x, y, z, t)}{\partial z}\right]^{2}\right\} \\
& \frac{\partial T_{02}(x, y, z, t)}{\partial t}=\alpha_{0 a s s}\left[\frac{\partial^{2} T_{02}(x, y, z, t)}{\partial x^{2}}+\frac{\partial^{2} T_{02}(x, y, z, t)}{\partial y^{2}}+\frac{\partial^{2} T_{02}(x, y, z, t)}{\partial z^{2}}\right]+\frac{\alpha_{0 a s s} T_{d}^{\varphi}}{T_{00}^{\varphi}(x, y, z, t)} \times \\
& \times\left[\frac{\partial^{2} T_{01}(x, y, z, t)}{\partial x^{2}}+\frac{\partial^{2} T_{01}(x, y, z, t)}{\partial y^{2}}+\frac{\partial^{2} T_{01}(x, y, z, t)}{\partial z^{2}}\right]-\frac{\varphi \alpha_{0 a s s} T_{d}^{\varphi}}{T_{00}^{\varphi+1}(x, y, z, t)}\left[\frac{\partial T_{00}(x, y, z, t)}{\partial x} \times\right. \\
& \left.\times \frac{\partial T_{01}(x, y, z, t)}{\partial x}+\frac{\partial T_{00}(x, y, z, t)}{\partial y} \frac{\partial T_{01}(x, y, z, t)}{\partial y}+\frac{\partial T_{00}(x, y, z, t)}{\partial z} \frac{\partial T_{01}(x, y, z, t)}{\partial z}\right] \\
& \frac{\partial T_{11}(x, y, z, t)}{\partial t}=\alpha_{0 a s s} \frac{\partial^{2} T_{11}(x, y, z, t)}{\partial x^{2}}+\alpha_{0 a s s} \frac{T_{01}(x, y, z, t)}{T_{00}(x, y, z, t)}\left\{g_{T}(x, y, z, T) \frac{\partial^{2} T_{00}(x, y, z, t)}{\partial x^{2}}+\right. \\
& \left.+g_{T}(x, y, z, T) \frac{\partial^{2} T_{00}(x, y, z, t)}{\partial y^{2}}+\frac{\partial}{\partial z}\left[g_{T}(x, y, z, T) \frac{\partial T_{00}(x, y, z, t)}{\partial z}\right]\right\}+\left\{\frac{\partial^{2} T_{01}(x, y, z, t)}{\partial x^{2}}+\right. \\
& +\frac{\partial}{\partial x}\left[g_{T}(x, y, z, T) \frac{\partial T_{01}(x, y, z, t)}{\partial x}\right]+\left[1+g_{T}(x, y, z, T)\right] \frac{\partial^{2} T_{01}(x, y, z, t)}{\partial y^{2}}+\left[1+g_{T}(x, y, z, T)\right] \times \\
& \left.\times \frac{\partial^{2} T_{01}(x, y, z, t)}{\partial z^{2}}\right\} \alpha_{0 a s s}+\left[\frac{T_{10}(x, y, z, t)}{T_{00}^{\varphi+1}(x, y, z, t)} \frac{\partial^{2} T_{00}(x, y, z, t)}{\partial x^{2}}+\frac{T_{10}(x, y, z, t)}{T_{00}^{\varphi+1}(x, y, z, t)} \frac{\partial^{2} T_{00}(x, y, z, t)}{\partial y^{2}}+\right. \\
& \left.+\frac{T_{10}(x, y, z, t)}{T_{00}^{\varphi+1}(x, y, z, t)} \frac{\partial^{2} T_{00}(x, y, z, t)}{\partial z^{2}}\right] \alpha_{0 a s s} T_{d}^{\varphi}+\frac{\alpha_{0 a s s} T_{d}^{\varphi}}{T_{00}^{\varphi}(x, y, z, t)}\left[\frac{\partial^{2} T_{10}(x, y, z, t)}{\partial x^{2}}+\frac{\partial^{2} T_{10}(x, y, z, t)}{\partial y^{2}}+\right. \\
& \left.+\frac{\partial^{2} T_{10}(x, y, z, t)}{\partial z^{2}}\right]+\frac{\alpha_{0 a s s} T_{d}^{\varphi}}{T_{00}^{\varphi}(x, y, z, t)}\left\{g_{T}(x, y, z, T) \frac{\partial^{2} T_{10}(x, y, z, t)}{\partial x^{2}}+g_{T}(x, y, z, T) \times\right. \\
& \left.\times \frac{\partial^{2} T_{10}(x, y, z, t)}{\partial y^{2}}+\frac{\partial}{\partial z}\left[g_{T}(x, y, z, T) \frac{\partial T_{00}(x, y, z, t)}{\partial z}\right]\right\}-\left[\frac{\partial T_{10}(x, y, z, t)}{\partial x} \frac{\partial T_{00}(x, y, z, t)}{\partial x}+\right. \\
& \left.+\frac{\partial T_{10}(x, y, z, t)}{\partial y} \frac{\partial T_{00}(x, y, z, t)}{\partial y}+\frac{\partial T_{10}(x, y, z, t)}{\partial z} \frac{\partial T_{00}(x, y, z, t)}{\partial z}\right] \frac{\varphi \alpha_{0 a s s} T_{d}^{\varphi}}{T_{00}^{\varphi+1}(x, y, z, t)}- \\
& -\alpha_{0 a s s} \varphi T_{d}^{\varphi} \frac{g_{T}(x, y, z, T)}{T_{00}^{\varphi+1}(x, y, z, t)}\left\{\left[\frac{\partial T_{00}(x, y, z, t)}{\partial x}\right]^{2}+\left[\frac{\partial T_{00}(x, y, z, t)}{\partial y}\right]^{2}+\left[\frac{\partial T_{00}(x, y, z, t)}{\partial z}\right]^{2}\right\} .
\end{aligned}
$$

Conditions for the functions $T_{i j}(x, y, z, t)(i \geq 0, j \geq 0)$

$$
\begin{aligned}
& \left.\frac{\partial T_{i j}(x, y, z, t)}{\partial x}\right|_{x=0}=0,\left.\frac{\partial T_{i j}(x, y, z, t)}{\partial x}\right|_{x=L_{x}}=0,\left.\frac{\partial T_{i j}(x, y, z, t)}{\partial y}\right|_{y=0}=0,\left.\frac{\partial T_{i j}(x, y, z, t)}{\partial y}\right|_{x=L_{y}}=0, \\
& \left.\frac{\partial T_{i j}(x, y, z, t)}{\partial z}\right|_{z=0}=0,\left.\frac{\partial T_{i j}(x, y, z, t)}{\partial z}\right|_{x=L_{z}}=0, T_{00}(x, y, z, 0)=f_{T}(x, y, z), T_{i j}(x, y, z, 0)=0, i \geq 1, j \geq 1 .
\end{aligned}
$$


Solutions of the above equations with account boundary and initial conditions for them have been calculated by using standard Fourier approach [31,32]. Using the approach leads to the following result

$$
\begin{aligned}
& T_{00}(x, y, z, t)=\frac{1}{L_{x} L_{y} L_{z}} \int_{0}^{L_{x}} \int_{0}^{L_{y}} \int_{0}^{L_{z}} f_{T}(u, v, w) d w d v d u+\frac{2}{L_{x} L_{y} L_{z}} \sum_{n=1}^{\infty} c_{n}(x) c_{n}(y) c_{n}(z) e_{n T}(t) \times \\
\times & \int_{0}^{L_{x}} c_{n}(u) \int_{0}^{L_{y}} c_{n}(v) \int_{0}^{L_{z}} c_{n}(w) f_{T}(u, v, w) d w d v d u+\frac{1}{L_{x} L_{y} L_{z}} \int_{0}^{t} \int_{0}^{L_{x}} \int_{0}^{L_{y}} \int_{0}^{L_{z}} \frac{p(u, v, w, \tau)}{v_{a s s}} d w d v d u d \tau+ \\
+ & \frac{2}{L_{x} L_{y} L_{z}} \sum_{n=1}^{\infty} c_{n}(x) c_{n}(y) c_{n}(z) e_{n T}(t) \int_{0}^{t} e_{n T}(-\tau) \int_{0}^{L_{x}} c_{n}(u) \int_{0}^{L_{y}} c_{n}(v) \int_{0}^{L_{z}} c_{n}(w) \frac{p(u, v, w, \tau)}{v_{a s s}} d w d v d u d \tau,
\end{aligned}
$$

where $c_{n}(\chi)=\cos (\pi n \chi / L), e_{n T}(t)=\exp \left[-\pi^{2} n^{2} \alpha_{0 a s s} t\left(\frac{1}{L_{x}^{2}}+\frac{1}{L_{y}^{2}}+\frac{1}{L_{z}^{2}}\right)\right]$;

$$
\begin{gathered}
T_{i 0}(x, y, z, t)=2 \frac{\pi \alpha_{0 a s s}}{L_{x}^{2} L_{y} L_{z}} \sum_{n=1}^{\infty} n c_{n}(x) c_{n}(y) c_{n}(z) e_{n T}(t) \int_{0}^{t} e_{n T}(-\tau) \int_{0}^{L_{x}} s_{n}(u) \int_{0}^{L_{y}} c_{n}(v) \int_{0}^{L_{z}} g_{T}(u, v, w, T) \times \\
\times c_{n}(w) \frac{\partial T_{i-10}(u, v, w, \tau)}{\partial u} d w d v d u d \tau+2 \frac{\pi \alpha_{0 a s s}}{L_{x} L_{y}^{2} L_{z}} \sum_{n=1}^{\infty} n c_{n}(x) c_{n}(y) c_{n}(z) e_{n T}(t) \int_{0}^{t} e_{n T}(-\tau) \times \\
\times \int_{0}^{L_{x}} c_{n}(u) \int_{0}^{L_{y}} s_{n}(v) \int_{0}^{L_{z}} g_{T}(u, v, w, T) c_{n}(w) \frac{\partial T_{i-10}(u, v, w, \tau)}{\partial v} d w d v d u d \tau+2 \frac{\pi \alpha_{0 a s s}}{L_{x} L_{y} L_{z}^{2}} \sum_{n=1}^{\infty} n c_{n}(x) c_{n}(y) \times \\
\times c_{n}(z) e_{n T}(t) \int_{0}^{t} e_{n T}(-\tau) \int_{0}^{L_{x}} c_{n}(u) \int_{0}^{L_{y}} c_{n}(v) \int_{0}^{L_{z}} s_{n}(w) g_{T}(u, v, w, T) \frac{\partial T_{i-10}(u, v, w, \tau)}{\partial w} d w d v d u d \tau, i \geq 1,
\end{gathered}
$$

where $s_{n}(\chi)=\sin (\pi n \chi / L)$;

$$
\begin{gathered}
T_{01}(x, y, z, t)=\alpha_{0 a s s} \frac{2 \pi T_{d}^{\varphi}}{L_{x}^{2} L_{y} L_{z}} \sum_{n=1}^{\infty} n c_{n}(x) c_{n}(y) c_{n}(z) e_{n T}(t) \int_{0}^{t} e_{n T}(-\tau) \int_{0}^{L_{x}} s_{n}(u) \int_{0}^{L_{y}} c_{n}(v) \int_{0}^{L_{z}} c_{n}(w) \times \\
\times \frac{\partial^{2} T_{00}(u, v, w, \tau)}{\partial u^{2}} \frac{d w d v d u d \tau}{T_{00}^{\varphi}(u, v, w, \tau)}+\alpha_{0 a s s} \frac{2 \pi T_{d}^{\varphi}}{L_{x} L_{y}^{2} L_{z}} \sum_{n=1}^{\infty} n c_{n}(x) c_{n}(y) c_{n}(z) e_{n T}(t) \int_{0}^{t} e_{n T}(-\tau) \times \\
\times \int_{0}^{L_{x}} c_{n}(u) \int_{0}^{L_{y}} s_{n}(v) \int_{0}^{L_{z}} c_{n}(w) \frac{\partial^{2} T_{00}(u, v, w, \tau)}{\partial v^{2}} \frac{d w d v d u d \tau}{T_{00}^{\varphi}(u, v, w, \tau)}+\alpha_{0 a s s} \frac{2 \pi T_{d}^{\varphi}}{L_{x} L_{y} L_{z}^{2}} \sum_{n=1}^{\infty} n c_{n}(x) c_{n}(y) c_{n}(z) \times \\
\times e_{n T}(t) \int_{0}^{t} e_{n T}(-\tau) \int_{0}^{L_{x}} c_{n}(u) \int_{0}^{L_{y}} c_{n}(v) \int_{0}^{L_{z}} s_{n}(w) \frac{\partial^{2} T_{00}(u, v, w, \tau)}{\partial u^{2}} \frac{d w d v d u d \tau}{T_{00}^{\varphi}(u, v, w, \tau)}-2 \frac{\varphi \alpha_{0 a s s}}{L_{x} L_{y} L_{z} \sum_{n=1}^{\infty} c_{n}(x) \times} \\
\times T_{d}^{\varphi} c_{n}(y) c_{n}(z) e_{n T}(t) \int_{0}^{t} e_{n T}(-\tau) \int_{0}^{L_{x}} c_{n}(u) \int_{0}^{L_{y}} c_{n}(v) \int_{0}^{L_{z}} c_{n}(w)\left[\frac{\partial T_{00}(u, v, w, \tau)}{\partial u}\right]^{2} \frac{d w d v d u d \tau}{T_{00}^{\varphi+1}(u, v, w, \tau)}- \\
-2 \varphi \frac{\alpha_{0 a s s} T_{d}^{\varphi}}{L_{x} L_{y} L_{z}} \sum_{n=1}^{\infty} c_{n}(x) c_{n}(y) c_{n}(z) e_{n T}(t) \int_{0}^{t} e_{n T}(-\tau) \int_{0}^{L_{x}} c_{n}(u) \int_{0}^{L_{y}} c_{n}(v) \int_{0}^{L_{z}} c_{n}(w)\left[\frac{\partial T_{00}(u, v, w, \tau)}{\partial v}\right]^{2} \times \\
\times \frac{d w d v d u d \tau}{T_{00}^{\varphi+1}(u, v, w, \tau)}-2 T_{d}^{\varphi} \frac{\varphi \alpha_{0 a s s} \sum_{n}^{\infty} c_{n}(x) c_{n}(y) c_{n}(z) e_{n T}(t) \int_{0}^{t} e_{n T}(-\tau) \int_{0}^{L_{x}} c_{n}(u) \int_{0}^{L_{y}} c_{n}(v) \times}{L_{x} L_{y} L_{z} \times 1} \\
\times \int_{0}^{L_{z}} c_{n}(w)\left[\frac{\partial T_{00}(u, v, w, \tau)}{\partial w} \frac{d w d v d u d \tau}{T_{00}^{\varphi+1}(u, v, w, \tau)} ;\right.
\end{gathered}
$$




$$
\begin{aligned}
& T_{02}(x, y, z, t)=\alpha_{0 a s s} \frac{2 \pi T_{d}^{\varphi}}{L_{x}^{2} L_{y} L_{z}} \sum_{n=1}^{\infty} n c_{n}(x) c_{n}(y) c_{n}(z) e_{n T}(t) \int_{0}^{t} e_{n T}(-\tau) \int_{0}^{L_{x}} s_{n}(u) \int_{0}^{L_{y}} c_{n}(v) \int_{0}^{L_{z}} c_{n}(w) \times \\
& \times \\
& \times \frac{\partial^{2} T_{01}(u, v, w, \tau)}{\partial u^{2}} \frac{d w d v d u d \tau}{T_{00}^{\varphi}(u, v, w, \tau)}+\alpha_{0 a s s} \frac{2 \pi T_{d}^{\varphi}}{L_{x} L_{y}^{2} L_{z}} \sum_{n=1}^{\infty} c_{n}(x) c_{n}(y) c_{n}(z) e_{n T}(t) \int_{0}^{t} e_{n T}(-\tau) \int_{0}^{L_{x}} c_{n}(u) \times \\
& \times n \int_{0}^{L_{y}} s_{n}(v) \int_{0}^{L_{z}} c_{n}(w) \frac{\partial^{2} T_{01}(u, v, w, \tau)}{\partial v^{2}} \frac{d w d v d u d \tau}{T_{00}^{\varphi}(u, v, w, \tau)}+\alpha_{0 a s s} \frac{2 \pi T_{d}^{\varphi}}{L_{x} L_{y} L_{z}^{2}} \sum_{n=1}^{\infty} n c_{n}(x) c_{n}(y) e_{n T}(t) \times \\
& \times c_{n}(z) \int_{0}^{t} e_{n T}(-\tau) \int_{0}^{L_{x}} c_{n}(u) \int_{0}^{L_{y}} c_{n}(v) \int_{0}^{L_{z}} s_{n}(w) \frac{\partial^{2} T_{01}(u, v, w, \tau)}{\partial w^{2}} \frac{d w d v d u d \tau}{T_{00}^{\varphi}(u, v, w, \tau)}-2 T_{d}^{\varphi} \frac{\pi \alpha_{0 a s s}}{L_{x}^{2} L_{y} L_{z}} \times \\
& \quad \times \sum_{n=1}^{\infty} c_{n}(x) c_{n}(y) c_{n}(z) e_{n T}(t) \int_{0}^{t} e_{n T}(-\tau) \int_{0}^{L_{x}} c_{n}(u) \int_{0}^{L_{y}} c_{n}(v) \int_{0}^{L_{z}} \frac{\partial T_{00}(u, v, w, \tau)}{\partial u} \frac{\partial T_{01}(u, v, w, \tau)}{\partial u} \times \\
& \quad \times c_{n}(w) \frac{d w d v d u d \tau}{T_{00}^{\varphi+1}(u, v, w, \tau)}-2 T_{d}^{\varphi} \frac{\pi \alpha_{0 a s s}}{L_{x} L_{y}^{2} L_{z} c_{n=1} c_{n}(x) c_{n}(y) c_{n}(z) e_{n T}(t) \int_{0}^{t} e_{n T}(-\tau) \int_{0}^{L_{x}} c_{n}(u) \times} \\
& \quad \times \int_{0}^{L_{y}} c_{n}(v) \int_{0}^{L_{z}} c_{n}(w) \frac{\partial T_{00}(u, v, w, \tau)}{\partial v} \frac{\partial T_{01}(u, v, w, \tau)}{\partial v} \frac{d w d v d u d \tau}{T_{00}^{\varphi+1}(u, v, w, \tau)}-2 \pi \frac{T_{d}^{\varphi} \alpha_{0 a s s}}{L_{x} L_{y} L_{z}^{2}} \sum_{n=1}^{\infty} c_{n}(x) \times
\end{aligned}
$$

$\times c_{n}(y) c_{n}(z) e_{n T}(t) \int_{0}^{t} e_{n T}(-\tau) \int_{0}^{L_{x}} c_{n}(u) \int_{0}^{L_{y}} c_{n}(v) \int_{0}^{L_{z}} c_{n}(w) \frac{\partial T_{00}(u, v, w, \tau)}{\partial w} \frac{\partial T_{01}(u, v, w, \tau)}{\partial w} \frac{d w d v d u d \tau}{T_{00}^{\varphi+1}(u, v, w, \tau)}$

$T_{11}(x, y, z, t)=\frac{2 \alpha_{0 a s s}}{L_{x} L_{y} L_{z}} \sum_{n=1}^{\infty} c_{n}(x) c_{n}(y) c_{n}(z) e_{n T}(t) \int_{0}^{t} e_{n T}(-\tau) \int_{0}^{L_{x}} c_{n}(u) \int_{0}^{L_{y}} c_{n}(v) \int_{0}^{L_{z}} c_{n}(w)\left\{\frac{\partial^{2} T_{00}(u, v, w, \tau)}{\partial w^{2}} \times\right.$ $\left.\times g_{T}(u, v, w, T)+g_{T}(u, v, w, T) \frac{\partial^{2} T_{00}(u, v, w, \tau)}{\partial v^{2}}+\frac{\partial}{\partial w}\left[g_{T}(w) \frac{\partial T_{00}(u, v, w, \tau)}{\partial w}\right]\right\} \frac{T_{01}(u, v, w, \tau)}{T_{00}(u, v, w, \tau)} d w d v d u d \tau+$ $+\frac{2 \alpha_{0 a s s}}{L_{x} L_{y} L_{z}} \sum_{n=1}^{\infty} c_{n}(x) c_{n}(y) c_{n}(z) e_{n T}(t) \int_{0}^{t} e_{n T}(-\tau) \int_{0}^{L_{x}} c_{n}(u) \int_{0}^{L_{y}} c_{n}(v) \int_{0}^{L_{z}} c_{n}(w)\left\{\frac{\partial^{2} T_{01}(u, v, w, \tau)}{\partial u^{2}}+\frac{\partial^{2} T_{01}(u, v, w, \tau)}{\partial u^{2}} \times\right.$ $\left.\times\left[1+g_{T}(u, v, w, T)\right]+\left[1+g_{T}(u, v, w, T)\right] \frac{\partial^{2} T_{01}(u, v, w, \tau)}{\partial v^{2}}+\frac{\partial}{\partial w}\left[g_{T}(u, v, w, T) \frac{\partial T_{01}(u, v, w, \tau)}{\partial w}\right]\right\} d w d v d u d \tau+$ $\times+2 \frac{\alpha_{0 a s s} T_{d}^{\varphi}}{L_{x} L_{y} L_{z}} \sum_{n=1}^{\infty} c_{n}(x) c_{n}(y) c_{n}(z) e_{n T}(t) \int_{0}^{t} e_{n T}(-\tau) \int_{0}^{L_{x}} c_{n}(u) \int_{0}^{L_{y}} c_{n}(v) \int_{0}^{L_{z}}\left[\frac{T_{10}(u, v, w, \tau)}{T_{00}^{\varphi+1}(u, v, w, \tau)} \frac{\partial^{2} T_{00}(u, v, w, \tau)}{\partial u^{2}}+\right.$ $\left.+\frac{T_{10}(u, v, w, \tau)}{T_{00}^{\varphi+1}(u, v, w, \tau)} \frac{\partial^{2} T_{00}(u, v, w, \tau)}{\partial v^{2}}+\frac{T_{10}(u, v, w, \tau)}{T_{00}^{\varphi+1}(u, v, w, \tau)} \frac{\partial^{2} T_{00}(u, v, w, \tau)}{\partial w^{2}}\right] c_{n}(w) d w d v d u d \tau+2 \frac{\alpha_{0 a s s} T_{d}^{\varphi}}{L_{x} L_{y} L_{z}} \times$ $\times \sum_{n=1}^{\infty} e_{n T}(t) \int_{0}^{t} e_{n T}(-\tau) \int_{0}^{L_{x}} c_{n}(u) \int_{0}^{L_{y}} c_{n}(v) \int_{0}^{L_{z}} c_{n}(w)\left[\frac{\partial^{2} T_{10}(u, v, w, \tau)}{\partial u^{2}}+\frac{\partial^{2} T_{10}(u, v, w, \tau)}{\partial v^{2}}+\frac{\partial^{2} T_{10}(u, v, w, \tau)}{\partial w^{2}}\right] \times$ $\times \frac{d w d v d u d \tau}{T_{00}^{\varphi}(u, v, w, \tau)} c_{n}(x) c_{n}(y) c_{n}(z)+2 \frac{\alpha_{0 a s s} T_{d}^{\varphi}}{L_{x} L_{y} L_{z}} 2 \sum_{n=1}^{\infty} c_{n}(x) c_{n}(y) c_{n}(z) e_{n T}(t) \int_{0}^{t} e_{n T}(-\tau) \int_{0}^{L_{x}} c_{n}(u) \int_{0}^{L_{y}} c_{n}(v) \times$ $\times \int_{0}^{L_{\tau}} c_{n}(w)\left\{g_{T}(u, v, w, T) \frac{\partial^{2} T_{00}(u, v, w, \tau)}{\partial u^{2}}+\frac{\partial}{\partial w}\left[g_{T}(u, v, w, T) \frac{\partial T_{00}(u, v, w, \tau)}{\partial w}\right]+\frac{\partial^{2} T_{00}(u, v, w, \tau)}{\partial v^{2}} \times\right.$ $\left.\times g_{T}(u, v, w, T)\right\} \frac{d w d v d u d \tau}{T_{00}^{\varphi}(u, v, w, \tau)}-2 \varphi \frac{\alpha_{0 a s s} T_{d}^{\varphi}}{L_{x} L_{y} L_{z}} \sum_{n=1}^{\infty} c_{n}(x) c_{n}(y) c_{n}(z) e_{n T}(t) \int_{0}^{t} e_{n T}(-\tau) \int_{0}^{L_{x}} c_{n}(u) \int_{0}^{L_{y}} c_{n}(v) \times$ 


$$
\begin{aligned}
& \times \int_{0}^{L_{z}} c_{n}(w)\left[\frac{\partial T_{10}(u, v, w, \tau)}{\partial u} \frac{\partial T_{00}(u, v, w, \tau)}{\partial u}+\frac{\partial T_{10}(u, v, w, \tau)}{\partial v} \frac{\partial T_{00}(u, v, w, \tau)}{\partial v}+\frac{\partial T_{10}(u, v, w, \tau)}{\partial w} \times\right. \\
\times & \left.\frac{\partial T_{00}(u, v, w, \tau)}{\partial w}\right] \frac{d w d v d u d \tau}{T_{00}^{\varphi+1}(u, v, w, \tau)}-2 \varphi \frac{\alpha_{0 a s s} T_{d}^{\varphi}}{L_{x} L_{y} L_{z}} \sum_{n=1}^{\infty} c_{n}(x) c_{n}(y) c_{n}(z) e_{n T}(t) \int_{0}^{t} e_{n T}(-\tau) \int_{0}^{L_{x}} c_{n}(u) \iint_{0}^{L_{y}} c_{n}(v) \times \\
& \times \int_{0}^{L_{z}} c_{n}(w)\left\{\left[\frac{\partial T_{00}(u, v, w, \tau)}{\partial u}\right]^{2}+\left[\frac{\partial T_{00}(u, v, w, \tau)}{\partial v}\right]^{2}+\left[\frac{\partial T_{00}(u, v, w, \tau)}{\partial w}\right]^{2}\right\} \frac{d w d v d u d \tau}{T_{00}^{\varphi+1}(u, v, w, \tau)} .
\end{aligned}
$$

Equations for initial-order approximation of concentrations of point radiation defects $\tilde{I}_{000}(\chi, \eta, \phi, \vartheta)$ and $\tilde{V}_{000}(\chi, \eta, \phi, \vartheta)$, corrections for the approximation $\tilde{I}_{i j k}(\chi, \eta, \phi, \vartheta)$ and $\tilde{V}_{i j k}(\chi, \eta, \phi, \vartheta)(i \geq 1, j \geq 1, k \geq 1)$, boundary and initial conditions for them have been obtain by substitution of the series (13) into Eqs.(4) and conditions (5) and equating of coefficients at equal powers of parameters, which have been used in the series (13). The equations and conditions could be written as

$$
\begin{aligned}
& \frac{\partial \tilde{I}_{000}(\chi, \eta, \phi, \vartheta)}{\partial \vartheta}=\sqrt{\frac{D_{0 I}}{D_{0 V}}} \frac{\partial^{2} \tilde{I}_{000}(\chi, \eta, \phi, \vartheta)}{\partial \chi^{2}}+\sqrt{\frac{D_{0 I}}{D_{0 V}}} \frac{\partial^{2} \tilde{I}_{000}(\chi, \eta, \phi, \vartheta)}{\partial \eta^{2}}+\sqrt{\frac{D_{0 I}}{D_{0 V}}} \frac{\partial^{2} \widetilde{I}_{000}(\chi, \eta, \phi, \vartheta)}{\partial \phi^{2}} \\
& \frac{\partial \tilde{V}_{000}(\chi, \eta, \phi, \vartheta)}{\partial \vartheta}=\sqrt{\frac{D_{0 V}}{D_{0 I}}} \frac{\partial^{2} \tilde{V}_{000}(\chi, \eta, \phi, \vartheta)}{\partial \chi^{2}}+\sqrt{\frac{D_{0 V}}{D_{0 I}}} \frac{\partial^{2} \tilde{V}_{000}(\chi, \eta, \phi, \vartheta)}{\partial \eta^{2}}+\sqrt{\frac{D_{0 V}}{D_{0 I}}} \frac{\partial^{2} \tilde{V}_{000}(\chi, \eta, \phi, \vartheta)}{\partial \phi^{2}} ; \\
& \frac{\partial \tilde{I}_{i 00}(\chi, \vartheta)}{\partial \vartheta}=\sqrt{\frac{D_{0 I}}{D_{0 V}}} \frac{\partial^{2} \tilde{I}_{i 00}(\chi, \eta, \phi, \vartheta)}{\partial \chi^{2}}+\sqrt{\frac{D_{0 I}}{D_{0 V}}} \frac{\partial}{\partial \chi}\left[g_{I}(\chi, \eta, \phi, T) \frac{\partial \tilde{I}_{i-100}(\chi, \eta, \phi, \vartheta)}{\partial \chi}\right]+ \\
& +\sqrt{\frac{D_{0 I}}{D_{0 V}}} \frac{\partial^{2} \tilde{I}_{i 00}(\chi, \eta, \phi, \vartheta)}{\partial \eta^{2}}+\sqrt{\frac{D_{0 I}}{D_{0 V}}} \frac{\partial}{\partial \eta}\left[g_{I}(\chi, \eta, \phi, T) \frac{\partial \tilde{I}_{i-100}(\chi, \eta, \phi, \vartheta)}{\partial \eta}\right]+ \\
& +\sqrt{\frac{D_{0 I}}{D_{0 V}}} \frac{\partial^{2} \tilde{I}_{i 00}(\chi, \eta, \phi, \vartheta)}{\partial \phi^{2}}+\sqrt{\frac{D_{0 I}}{D_{0 V}}} \frac{\partial}{\partial \phi}\left[g_{I}(\chi, \eta, \phi, T) \frac{\partial \tilde{I}_{i-100}(\chi, \eta, \phi, \vartheta)}{\partial \phi}\right] \\
& \frac{\partial \tilde{V}_{i 00}(\chi, \vartheta)}{\partial \vartheta}=\sqrt{\frac{D_{0 V}}{D_{0 I}}} \frac{\partial^{2} \tilde{V}_{i 00}(\chi, \eta, \phi, \vartheta)}{\partial \chi^{2}}+\sqrt{\frac{D_{0 V}}{D_{0 I}}} \frac{\partial}{\partial \chi}\left[g_{V}(\chi, \eta, \phi, T) \frac{\partial \tilde{V}_{i-100}(\chi, \eta, \phi, \vartheta)}{\partial \chi}\right]+ \\
& +\sqrt{\frac{D_{0 V}}{D_{0 I}}} \frac{\partial^{2} \tilde{V}_{i 00}(\chi, \eta, \phi, \vartheta)}{\partial \eta^{2}}+\sqrt{\frac{D_{0 V}}{D_{0 I}}} \frac{\partial}{\partial \eta}\left[g_{V}(\chi, \eta, \phi, T) \frac{\partial \tilde{V}_{i-100}(\chi, \eta, \phi, \vartheta)}{\partial \eta}\right]+ \\
& +\sqrt{\frac{D_{0 V}}{D_{0 I}}} \frac{\partial^{2} \tilde{V}_{i 00}(\chi, \eta, \phi, \vartheta)}{\partial \phi^{2}}+\sqrt{\frac{D_{0 V}}{D_{0 I}}} \frac{\partial}{\partial \phi}\left[g_{V}(\chi, \eta, \phi, T) \frac{\partial \tilde{V}_{i-100}(\chi, \eta, \phi, \vartheta)}{\partial \phi}\right], i \geq 1 ; \\
& \frac{\partial \tilde{I}_{010}(\chi, \eta, \phi, \vartheta)}{\partial \vartheta}=\sqrt{\frac{D_{0 I}}{D_{0 V}}} \frac{\partial^{2} \widetilde{I}_{010}(\chi, \eta, \phi, \vartheta)}{\partial \chi^{2}}+\sqrt{\frac{D_{0 I}}{D_{0 V}}} \frac{\partial^{2} \widetilde{I}_{010}(\chi, \eta, \phi, \vartheta)}{\partial \eta^{2}}+ \\
& +\sqrt{\frac{D_{0 I}}{D_{0 V}}} \frac{\partial^{2} \tilde{I}_{010}(\chi, \eta, \phi, \vartheta)}{\partial \phi^{2}}-\left[1+\varepsilon_{I, V} g_{I, V}(\chi, \eta, \phi, T)\right] \tilde{I}_{000}(\chi, \eta, \phi, \vartheta) \tilde{V}_{000}(\chi, \eta, \phi, \vartheta) \\
& \frac{\partial \tilde{V}_{010}(\chi, \eta, \phi, \vartheta)}{\partial \vartheta}=\sqrt{\frac{D_{0 V}}{D_{0 I}}} \frac{\partial^{2} \tilde{V}_{010}(\chi, \eta, \phi, \vartheta)}{\partial \chi^{2}}+\sqrt{\frac{D_{0 V}}{D_{0 I}}} \frac{\partial^{2} \tilde{V}_{010}(\chi, \eta, \phi, \vartheta)}{\partial \eta^{2}}+ \\
& +\sqrt{\frac{D_{0 V}}{D_{0 I}}} \frac{\partial^{2} \tilde{V}_{010}(\chi, \eta, \phi, \vartheta)}{\partial \phi^{2}}-\left[1+\varepsilon_{I, V} g_{I, V}(\chi, \eta, \phi, T)\right] \tilde{I}_{000}(\chi, \eta, \phi, \vartheta) \tilde{V}_{000}(\chi, \eta, \phi, \vartheta) ; \\
& \frac{\partial \tilde{I}_{020}(\chi, \eta, \phi, \vartheta)}{\partial \vartheta}=\sqrt{\frac{D_{0 I}}{D_{0 V}}} \frac{\partial^{2} \widetilde{I}_{020}(\chi, \eta, \phi, \vartheta)}{\partial \chi^{2}}+\sqrt{\frac{D_{0 I}}{D_{0 V}}} \frac{\partial^{2} \widetilde{I}_{020}(\chi, \eta, \phi, \vartheta)}{\partial \eta^{2}}+\sqrt{\frac{D_{0 I}}{D_{0 V}}} \frac{\partial^{2} \tilde{I}_{020}(\chi, \eta, \phi, \vartheta)}{\partial \phi^{2}}-
\end{aligned}
$$


$-\left[1+\varepsilon_{I, V} g_{I, V}(\chi, \eta, \phi, T)\right]\left[\tilde{I}_{010}(\chi, \eta, \phi, \vartheta) \tilde{V}_{000}(\chi, \eta, \phi, \vartheta)+\tilde{I}_{000}(\chi, \eta, \phi, \vartheta) \tilde{V}_{010}(\chi, \eta, \phi, \vartheta)\right]$ $\frac{\partial \tilde{V}_{020}(\chi, \eta, \phi, \vartheta)}{\partial \vartheta}=\sqrt{\frac{D_{0 I}}{D_{0 V}}} \frac{\partial^{2} \tilde{V}_{020}(\chi, \eta, \phi, \vartheta)}{\partial \chi^{2}}+\sqrt{\frac{D_{0 I}}{D_{0 V}}} \frac{\partial^{2} \tilde{V}_{020}(\chi, \eta, \phi, \vartheta)}{\partial \eta^{2}}+\sqrt{\frac{D_{0 I}}{D_{0 V}}} \frac{\partial^{2} \tilde{V}_{020}(\chi, \eta, \phi, \vartheta)}{\partial \phi^{2}}-$

$-\left[1+\varepsilon_{I, V} g_{I, V}(\chi, \eta, \phi, T)\right]\left[\tilde{I}_{010}(\chi, \eta, \phi, \vartheta) \tilde{V}_{000}(\chi, \eta, \phi, \vartheta)+\tilde{I}_{000}(\chi, \eta, \phi, \vartheta) \tilde{V}_{010}(\chi, \eta, \phi, \vartheta)\right] ;$

$\frac{\partial \tilde{I}_{001}(\chi, \eta, \phi, \vartheta)}{\partial \vartheta}=\sqrt{\frac{D_{0 I}}{D_{0 V}}} \frac{\partial^{2} \widetilde{I}_{001}(\chi, \eta, \phi, \vartheta)}{\partial \chi^{2}}+\sqrt{\frac{D_{0 I}}{D_{0 V}}} \frac{\partial^{2} \widetilde{I}_{001}(\chi, \eta, \phi, \vartheta)}{\partial \eta^{2}}+$

$+\sqrt{\frac{D_{0 I}}{D_{0 V}}} \frac{\partial^{2} \tilde{I}_{001}(\chi, \eta, \phi, \vartheta)}{\partial \phi^{2}}-\left[1+\varepsilon_{I, I} g_{I, I}(\chi, \eta, \phi, T)\right] \tilde{I}_{000}^{2}(\chi, \eta, \phi, \vartheta)$

$\frac{\partial \tilde{V}_{001}(\chi, \eta, \phi, \vartheta)}{\partial \vartheta}=\sqrt{\frac{D_{0 V}}{D_{0 I}}} \frac{\partial^{2} \tilde{V}_{001}(\chi, \eta, \phi, \vartheta)}{\partial \chi^{2}}+\sqrt{\frac{D_{0 V}}{D_{0 I}}} \frac{\partial^{2} \tilde{V}_{001}(\chi, \eta, \phi, \vartheta)}{\partial \eta^{2}}+$

$+\sqrt{\frac{D_{0 V}}{D_{0 I}}} \frac{\partial^{2} \tilde{V}_{001}(\chi, \eta, \phi, \vartheta)}{\partial \phi^{2}}-\left[1+\varepsilon_{I, I} g_{I, I}(\chi, \eta, \phi, T)\right] \tilde{V}_{000}^{2}(\chi, \eta, \phi, \vartheta) ;$

$\frac{\partial \tilde{I}_{110}(\chi, \eta, \phi, \vartheta)}{\partial \vartheta}=\sqrt{\frac{D_{0 I}}{D_{0 V}}} \frac{\partial^{2} \tilde{I}_{110}(\chi, \eta, \phi, \vartheta)}{\partial \chi^{2}}+\sqrt{\frac{D_{0 I}}{D_{0 V}}} \frac{\partial}{\partial \chi}\left[g_{I}(\chi, \eta, \phi, T) \frac{\partial \tilde{I}_{010}(\chi, \eta, \phi, \vartheta)}{\partial \chi}\right]+\sqrt{\frac{D_{0 I}}{D_{0 V}}} \times$

$\times \frac{\partial^{2} \tilde{I}_{110}(\chi, \eta, \phi, \vartheta)}{\partial \eta^{2}}+\sqrt{\frac{D_{0 I}}{D_{0 V}}} \frac{\partial}{\partial \eta}\left[g_{I}(\chi, \eta, \phi, T) \frac{\partial \tilde{I}_{010}(\chi, \eta, \phi, \vartheta)}{\partial \eta}\right]+\sqrt{\frac{D_{0 I}}{D_{0 V}}} \frac{\partial^{2} \tilde{I}_{110}(\chi, \eta, \phi, \vartheta)}{\partial \phi^{2}}+$

$+\sqrt{\frac{D_{0 I}}{D_{0 V}}} \frac{\partial}{\partial \phi}\left[g_{I}(\chi, \eta, \phi, T) \frac{\partial \tilde{I}_{010}(\chi, \eta, \phi, \vartheta)}{\partial \phi}\right]-\left[1+\varepsilon_{I, I} g_{I, I}(\chi, \eta, \phi, T)\right] \times$

$\times\left[\tilde{I}_{100}(\chi, \eta, \phi, \vartheta) \tilde{V}_{000}(\chi, \eta, \phi, \vartheta)+\tilde{I}_{000}(\chi, \eta, \phi, \vartheta) \tilde{V}_{100}(\chi, \eta, \phi, \vartheta)\right]$

$\frac{\partial \tilde{V}_{110}(\chi, \eta, \phi, \vartheta)}{\partial \vartheta}=\sqrt{\frac{D_{0 V}}{D_{0 I}}} \frac{\partial^{2} \tilde{V}_{110}(\chi, \eta, \phi, \vartheta)}{\partial \chi^{2}}+\sqrt{\frac{D_{0 V}}{D_{0 I}}} \frac{\partial}{\partial \chi}\left[g_{V}(\chi, \eta, \phi, T) \frac{\partial \tilde{V}_{010}(\chi, \eta, \phi, \vartheta)}{\partial \chi}\right]+\sqrt{\frac{D_{0 V}}{D_{0 I}}} \times$

$\times \frac{\partial^{2} \tilde{V}_{110}(\chi, \eta, \phi, \vartheta)}{\partial \eta^{2}}+\sqrt{\frac{D_{0 V}}{D_{0 I}}} \frac{\partial}{\partial \eta}\left[g_{V}(\chi, \eta, \phi, T) \frac{\partial \tilde{V}_{010}(\chi, \eta, \phi, \vartheta)}{\partial \eta}\right]+\sqrt{\frac{D_{0 V}}{D_{0 I}}} \frac{\partial^{2} \tilde{V}_{110}(\chi, \eta, \phi, \vartheta)}{\partial \phi^{2}}+$

$+\sqrt{\frac{D_{0 V}}{D_{0 I}}} \frac{\partial}{\partial \phi}\left[g_{V}(\chi, \eta, \phi, T) \frac{\partial \tilde{V}_{010}(\chi, \eta, \phi, \vartheta)}{\partial \phi}\right]-\left[1+\varepsilon_{V, V} g_{V, V}(\chi, \eta, \phi, T)\right] \times$

$\times\left[\tilde{V}_{100}(\chi, \eta, \phi, \vartheta) \tilde{I}_{000}(\chi, \eta, \phi, \vartheta)+\tilde{V}_{000}(\chi, \eta, \phi, \vartheta) \tilde{I}_{100}(\chi, \eta, \phi, \vartheta)\right] ;$

$\frac{\partial \tilde{I}_{002}(\chi, \eta, \phi, \vartheta)}{\partial \vartheta}=\sqrt{\frac{D_{0 I}}{D_{0 V}}} \frac{\partial^{2} \widetilde{I}_{002}(\chi, \eta, \phi, \vartheta)}{\partial \chi^{2}}+\sqrt{\frac{D_{0 I}}{D_{0 V}}} \frac{\partial^{2} \tilde{I}_{002}(\chi, \eta, \phi, \vartheta)}{\partial \eta^{2}}+\sqrt{\frac{D_{0 I}}{D_{0 V}}} \frac{\partial^{2} \tilde{I}_{002}(\chi, \eta, \phi, \vartheta)}{\partial \phi^{2}}-$

$-\left[1+\varepsilon_{I, I} g_{I, I}(\chi, \eta, \phi, T)\right] \tilde{I}_{001}(\chi, \eta, \phi, \vartheta) \tilde{I}_{000}(\chi, \eta, \phi, \vartheta)$

$\frac{\partial \tilde{V}_{002}(\chi, \eta, \phi, \vartheta)}{\partial \vartheta}=\sqrt{\frac{D_{0 V}}{D_{0 I}}} \frac{\partial^{2} \tilde{V}_{002}(\chi, \eta, \phi, \vartheta)}{\partial \chi^{2}}+\sqrt{\frac{D_{0 V}}{D_{0 I}}} \frac{\partial^{2} \tilde{V}_{002}(\chi, \eta, \phi, \vartheta)}{\partial \eta^{2}}+\sqrt{\frac{D_{0 V}}{D_{0 I}}} \frac{\partial^{2} \tilde{V}_{002}(\chi, \eta, \phi, \vartheta)}{\partial \phi^{2}}-$

$-\left[1+\varepsilon_{V, V} g_{V, V}(\chi, \eta, \phi, E)\right] \tilde{V}_{001}(\chi, \eta, \phi, \vartheta) \tilde{V}_{000}(\chi, \eta, \phi, \vartheta) ;$

$\frac{\partial \tilde{I}_{101}(\chi, \eta, \phi, \vartheta)}{\partial \vartheta}=\sqrt{\frac{D_{0 I}}{D_{0 V}}} \frac{\partial^{2} \tilde{I}_{101}(\chi, \eta, \phi, \vartheta)}{\partial \chi^{2}}+\frac{\partial}{\partial \chi}\left[g_{I}(\chi, \eta, \phi, T) \frac{\partial \tilde{I}_{001}(\chi, \eta, \phi, \vartheta)}{\partial \chi}\right] \sqrt{\frac{D_{0 I}}{D_{0 V}}}+\sqrt{\frac{D_{0 I}}{D_{0 V}}} \times$

$\times \frac{\partial^{2} \tilde{I}_{101}(\chi, \eta, \phi, \vartheta)}{\partial \eta^{2}}+\sqrt{\frac{D_{0 I}}{D_{0 V}}} \frac{\partial}{\partial \eta}\left[g_{I}(\chi, \eta, \phi, T) \frac{\partial \tilde{I}_{001}(\chi, \eta, \phi, \vartheta)}{\partial \eta}\right]+\sqrt{\frac{D_{0 I}}{D_{0 V}}} \frac{\partial^{2} \tilde{I}_{101}(\chi, \eta, \phi, \vartheta)}{\partial \phi^{2}}+$ 


$$
\begin{aligned}
& +\sqrt{\frac{D_{0 I}}{D_{0 V}}} \frac{\partial}{\partial \phi}\left[g_{I}(\chi, \eta, \phi, T) \frac{\partial \tilde{I}_{001}(\chi, \eta, \phi, \vartheta)}{\partial \phi}\right]-\left[1+\varepsilon_{I} g_{I}(\chi, \eta, \phi, T)\right] \tilde{I}_{100}(\chi, \eta, \phi, \vartheta) \tilde{V}_{000}(\chi, \eta, \phi, \vartheta) \\
& \frac{\partial \tilde{V}_{101}(\chi, \eta, \phi, \vartheta)}{\partial \vartheta}=\sqrt{\frac{D_{0 V}}{D_{0 I}}} \frac{\partial^{2} \tilde{V}_{101}(\chi, \eta, \phi, \vartheta)}{\partial \chi^{2}}+\frac{\partial}{\partial \chi}\left[g_{V}(\chi, \eta, \phi, T) \frac{\partial \tilde{V}_{001}(\chi, \eta, \phi, \vartheta)}{\partial \chi}\right] \sqrt{\frac{D_{0 V}}{D_{0 I}}}+\sqrt{\frac{D_{0 V}}{D_{0 I}}} \times \\
& \times \frac{\partial^{2} \tilde{V}_{101}(\chi, \eta, \phi, \vartheta)}{\partial \eta^{2}}+\sqrt{\frac{D_{0 V}}{D_{0 I}}} \frac{\partial}{\partial \eta}\left[g_{V}(\chi, \eta, \phi, T) \frac{\partial \tilde{V}_{001}(\chi, \eta, \phi, \vartheta)}{\partial \eta}\right]+\sqrt{\frac{D_{0 V}}{D_{0 I}}} \frac{\partial^{2} \tilde{V}_{101}(\chi, \eta, \phi, \vartheta)}{\partial \phi^{2}}+ \\
& +\sqrt{\frac{D_{0 V}}{D_{0 I}}} \frac{\partial}{\partial \phi}\left[g_{V}(\chi, \eta, \phi, T) \frac{\partial \tilde{V}_{001}(\chi, \eta, \phi, \vartheta)}{\partial \phi}\right]-\left[1+\varepsilon_{V} g_{V}(\chi, \eta, \phi, T)\right] \tilde{I}_{000}(\chi, \eta, \phi, \vartheta) \tilde{V}_{100}(\chi, \eta, \phi, \vartheta) ;
\end{aligned}
$$

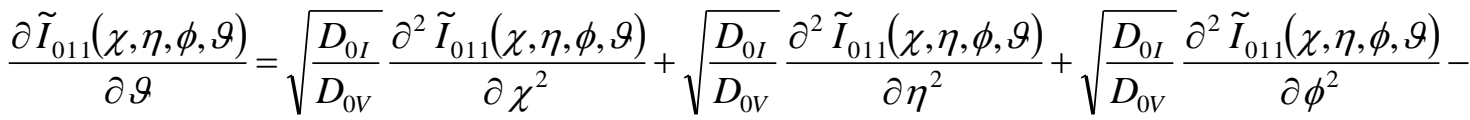

$$
\begin{aligned}
& -\left[1+\varepsilon_{I, I} g_{I, I}(\chi, \eta, \phi, T)\right] \tilde{I}_{000}(\chi, \eta, \phi, \vartheta) \tilde{I}_{010}(\chi, \eta, \phi, \vartheta)- \\
& -\left[1+\varepsilon_{I, V} g_{I, V}(\chi, \eta, \phi, T)\right] \tilde{I}_{001}(\chi, \eta, \phi, \vartheta) \tilde{V}_{000}(\chi, \eta, \phi, \vartheta) \\
& \frac{\partial \tilde{V}_{011}(\chi, \eta, \phi, \vartheta)}{\partial \vartheta}=\sqrt{\frac{D_{0 V}}{D_{0 I}}} \frac{\partial^{2} \tilde{V}_{011}(\chi, \eta, \phi, \vartheta)}{\partial \chi^{2}}+\sqrt{\frac{D_{0 V}}{D_{0 I}}} \frac{\partial^{2} \tilde{V}_{011}(\chi, \eta, \phi, \vartheta)}{\partial \eta^{2}}+\sqrt{\frac{D_{0 V}}{D_{0 I}}} \frac{\partial^{2} \tilde{V}_{011}(\chi, \eta, \phi, \vartheta)}{\partial \phi^{2}}- \\
& -\left[1+\varepsilon_{V, V} g_{V, V}(\chi, \eta, \phi, T)\right] \tilde{V}_{000}(\chi, \eta, \phi, \vartheta) \tilde{V}_{010}(\chi, \eta, \phi, \vartheta)- \\
& -\left[1+\varepsilon_{I, V} g_{I, V}(\chi, \eta, \phi, t)\right] \tilde{I}_{000}(\chi, \eta, \phi, \vartheta) \tilde{V}_{001}(\chi, \eta, \phi, \vartheta) ; \\
& \left.\frac{\partial \tilde{\rho}_{i j k}(\chi, \eta, \phi, \vartheta)}{\partial \chi}\right|_{x=0}=0,\left.\frac{\partial \tilde{\rho}_{i j k}(\chi, \eta, \phi, \vartheta)}{\partial \chi}\right|_{x=1}=0,\left.\frac{\partial \tilde{\rho}_{i j k}(\chi, \eta, \phi, \vartheta)}{\partial \eta}\right|_{\eta=0}=0,\left.\frac{\partial \tilde{\rho}_{i j k}(\chi, \eta, \phi, \vartheta)}{\partial \eta}\right|_{\eta=1}=0 \text {, } \\
& \left.\frac{\partial \tilde{\rho}_{i j k}(\chi, \eta, \phi, \vartheta)}{\partial \phi}\right|_{\phi=0}=0,\left.\frac{\partial \tilde{\rho}_{i j k}(\chi, \eta, \phi, \vartheta)}{\partial \phi}\right|_{\phi=1}=0(i \geq 0, j \geq 0, k \geq 0) ; \tilde{\rho}_{000}(\chi, \eta, \phi, 0)=f_{\rho}(\chi, \eta, \phi) / \rho^{*}, \\
& \tilde{\rho}_{i j k}(\chi, \eta, \phi, 0)=0(i \geq 0, j \geq 0, k \geq 0) \text {. }
\end{aligned}
$$

Solutions of the above equations with account boundary and initial conditions for them have been calculated by using standard Fourier approach $[31,32]$. Using the approach leads to the following result

$$
\tilde{\rho}_{000}(\chi, \eta, \phi, \vartheta)=\frac{1}{L}+\frac{2}{L} \sum_{n=1}^{\infty} F_{n \rho} c(\chi) c(\eta) c(\phi) e_{n \rho}(\vartheta),
$$

where $F_{n \rho}=\frac{1}{\rho^{*}} \int_{0}^{1} \cos (\pi n u) \int_{0}^{1} \cos (\pi n v) \int_{0}^{1} \cos (\pi n w) f_{n \rho}(u, v, w) d w d v d u, e_{n I}(\vartheta)=\exp \left(-\pi^{2} n^{2} \vartheta \sqrt{D_{0 V} / D_{0 I}}\right)$,

$$
\begin{aligned}
e_{n V}(\vartheta) & =\exp \left(-\pi^{2} n^{2} \vartheta \sqrt{D_{0 I} / D_{0 V}}\right), c_{n}(\chi)=\cos (\pi n \chi) ; \\
& \tilde{I}_{i 00}(\chi, \eta, \phi, \vartheta)=-2 \pi \sqrt{\frac{D_{0 I}}{D_{0 V}}} \sum_{n=1}^{\infty} c_{n}(\chi) c(\eta) c(\phi) e_{n I}(\vartheta) \int_{0}^{\vartheta} e_{n I}(-\tau) \int_{0}^{1} s_{n}(u) \int_{0}^{1} c_{n}(v) \int_{0}^{1} g_{I}(u, v, w, T) \times \\
& \times n c_{n}(w) \frac{\partial \tilde{I}_{i-100}(u, v, w, \tau)}{\partial u} d w d v d u d \tau-2 \pi \sqrt{\frac{D_{0 I}}{D_{0 V}} \sum_{n=1}^{\infty} n c_{n}(\chi) c(\eta) c(\phi) e_{n I}(\vartheta) \int_{0}^{\vartheta} e_{n I}(-\tau) \times} \\
& \times \int_{0}^{1} c_{n}(u) \int_{0}^{1} s_{n}(v) \int_{0}^{1} c_{n}(w) g_{I}(u, v, w, T) \frac{\partial \tilde{I}_{i-100}(u, v, w, \tau)}{\partial v} d w d v d u d \tau-2 \pi \sqrt{\frac{D_{0 I}}{D_{0 V}}} \sum_{n=1}^{\infty} n c_{n}(\chi) \times \\
& \times c(\eta) c(\phi) e_{n I}(\vartheta) \int_{0}^{\vartheta} e_{n I}(-\tau) \int_{0}^{1} c_{n}(u) \int_{0}^{1} c_{n}(v) \int_{0}^{1} s_{n}(w) g_{I}(u, v, w, T) \frac{\partial \tilde{I}_{i-100}(u, v, w, \tau)}{\partial w} d w d v d u d \tau
\end{aligned}
$$




$$
\begin{aligned}
& \tilde{V}_{i 00}(\chi, \eta, \phi, \vartheta)=-2 \pi \sqrt{\frac{D_{0 V}}{D_{0 I}}} \sum_{n=1}^{\infty} n c_{n}(\chi) c(\eta) c(\phi) e_{n V}(\vartheta) \int_{0}^{\vartheta} e_{n I}(-\tau) \int_{0}^{1} s_{n}(u) \int_{0}^{1} c_{n}(v) \int_{0}^{1} g_{V}(u, v, w, T) \times \\
& \times n c_{n}(w) \frac{\partial \tilde{V}_{i-100}(u, \tau)}{\partial u} d w d v d u d \tau-2 \pi \sqrt{\frac{D_{0 V}}{D_{0 I}}} \sum_{n=1}^{\infty} n c_{n}(\chi) c(\eta) c(\phi) e_{n V}(\vartheta) \int_{0}^{\vartheta} e_{n I}(-\tau) \int_{0}^{1} c_{n}(u) \times \\
& \times \int_{0}^{1} s_{n}(v) \int_{0}^{1} c_{n}(w) g_{V}(u, v, w, T) \frac{\partial \tilde{V}_{i-100}(u, \tau)}{\partial v} d w d v d u d \tau-2 \pi \sqrt{\frac{D_{0 V}}{D_{0 I}}} \sum_{n=1}^{\infty} c_{n}(\chi) c(\eta) c(\phi) e_{n V}(\vartheta) \times \\
& \times n \int_{0}^{\vartheta} e_{n I}(-\tau) \int_{0}^{1} c_{n}(u) \int_{0}^{1} c_{n}(v) \int_{0}^{1} s_{n}(w) g_{V}(u, v, w, T) \frac{\partial \tilde{V}_{i-100}(u, \tau)}{\partial w} d w d v d u d \tau, i \geq 1,
\end{aligned}
$$

where $s_{n}(\chi)=\sin (\pi n \chi)$;

$$
\begin{gathered}
\tilde{\rho}_{010}(\chi, \eta, \phi, \vartheta)=-2 \sum_{n=1}^{\infty} c_{n}(\chi) c_{n}(\eta) c_{n}(\phi) e_{n \rho}(\vartheta) \int_{0}^{\vartheta} e_{n \rho}(-\tau) \int_{0}^{1} c_{n}(u) \int_{0}^{1} c_{n}(v) \int_{0}^{1} c_{n}(w) \times \\
\times\left[1+\varepsilon_{I, V} g_{I, V}(u, v, w, T)\right] \tilde{I}_{000}(u, v, w, \tau) \tilde{V}_{000}(u, v, w, \tau) d w d v d u d \tau ; \\
\tilde{\rho}_{020}(\chi, \eta, \phi, \vartheta)=-2 \sqrt{\frac{D_{0 I}}{D_{0 V}}} \sum_{n=1}^{\infty} c_{n}(\chi) c_{n}(\eta) c_{n}(\phi) e_{n \rho}(\vartheta) \int_{0}^{\vartheta} e_{n \rho}(-\tau) \int_{000}^{11} \int_{00}^{11}\left[1+\varepsilon_{I, V} g_{I, V}(u, v, w, T)\right] \times \\
\times c_{n}(u) c_{n}(v) c_{n}(w)\left[\tilde{I}_{010}(u, v, w, \tau) \tilde{V}_{000}(u, v, w, \tau)+\tilde{I}_{000}(u, v, w, \tau) \tilde{V}_{010}(u, v, w, \tau)\right] d w d v d u d \tau ; \\
\tilde{\rho}_{001}(\chi, \eta, \phi, \vartheta)=-2 \sum_{n=1}^{\infty} c_{n}(\chi) c_{n}(\eta) c_{n}(\phi) e_{n \rho}(\vartheta) \int_{0}^{\vartheta} e_{n \rho}(-\tau) \int_{0}^{1} c_{n}(u) \int_{0}^{1} c_{n}(v) \int_{0}^{1} c_{n}(w) \times \\
\times\left[1+\varepsilon_{\rho, \rho} g_{\rho, \rho}(u, v, w, T)\right] \tilde{\rho}_{000}^{2}(u, v, w, \tau) d w d v d u d \tau ;
\end{gathered}
$$

$\tilde{\rho}_{002}(\chi, \eta, \phi, \vartheta)=-2 \sum_{n=1}^{\infty} c_{n}(\chi) c_{n}(\eta) c_{n}(\phi) e_{n \rho}(\vartheta) \int_{0}^{\vartheta} e_{n \rho}(-\tau) \int_{0}^{1} c_{n}(u) \int_{0}^{1} c_{n}(v) \int_{0}^{1} c_{n}(w) \times$

$\times\left[1+\varepsilon_{\rho, \rho} g_{\rho, \rho}(u, v, w, T)\right] \tilde{\rho}_{001}(u, v, w, \tau) \tilde{\rho}_{000}(u, v, w, \tau) d w d v d u d \tau ;$

$$
\begin{aligned}
& \tilde{I}_{110}(\chi, \eta, \phi, \vartheta)=-2 \pi \sqrt{\frac{D_{0 I}}{D_{0 V}}} \sum_{n=1}^{\infty} n c_{n}(\chi) c_{n}(\eta) c_{n}(\phi) e_{n I}(\vartheta) \int_{0}^{\vartheta} e_{n I}(-\tau) \int_{0}^{1} s_{n}(u) \int_{0}^{1} c_{n}(v) \int_{0}^{1} c_{n}(u) \times \\
& \times g_{I}(u, v, w, T) \frac{\partial \tilde{I}_{i-100}(u, v, w, \tau)}{\partial u} d w d v d u d \tau-2 \pi \sqrt{\frac{D_{0 I}}{D_{0 V}} \sum_{n=1}^{\infty} n c_{n}(\chi) c_{n}(\eta) c_{n}(\phi) e_{n I}(\vartheta) \times}
\end{aligned}
$$$$
\times \int_{0}^{\vartheta} e_{n I}(-\tau) \int_{0}^{1} c_{n}(u) \int_{0}^{1} s_{n}(v) \int_{0}^{1} c_{n}(u) g_{I}(u, v, w, T) \frac{\partial \tilde{I}_{i-100}(u, v, w, \tau)}{\partial v} d w d v d u d \tau-2 \pi \sqrt{\frac{D_{0 I}}{D_{0 V}}} \times
$$$$
\times \sum_{n=1}^{\infty} n e_{n I}(\vartheta) \int_{0}^{\vartheta} e_{n I}(-\tau) \int_{0}^{1} c_{n}(u) \int_{0}^{1} c_{n}(v) \int_{0}^{1} s_{n}(u) g_{I}(u, v, w, T) \frac{\partial \tilde{I}_{i-100}(u, v, w, \tau)}{\partial w} d w d v d u d \tau \times
$$$$
\times c_{n}(\chi) c_{n}(\eta) c_{n}(\phi)-2 \sum_{n=1}^{\infty} c_{n}(\chi) e_{n I}(\vartheta) c_{n}(\eta) c_{n}(\phi) \int_{0}^{\vartheta} e_{n I}(-\tau) \int_{0}^{1} c_{n}(u) \int_{0}^{1} c_{n}(v) \int_{0}^{1}\left[\tilde{I}_{100}(u, v, w, \tau) \times\right.
$$$$
\left.\times \tilde{V}_{000}(u, v, w, \tau)+\tilde{I}_{000}(u, v, w, \tau) \tilde{V}_{100}(u, v, w, \tau)\right]\left[1+\varepsilon_{I, V} g_{I, V}(u, v, w, T)\right] c_{n}(v) d w d v d u d \tau
$$$$
\tilde{V}_{110}(\chi, \eta, \phi, \vartheta)=-2 \pi \sqrt{\frac{D_{0 V}}{D_{0 I}}} \sum_{n=1}^{\infty} n c_{n}(\chi) c_{n}(\eta) c_{n}(\phi) e_{n V}(\vartheta) \int_{0}^{\vartheta} e_{n V}(-\tau) \int_{0}^{1} s_{n}(u) \int_{0}^{1} c_{n}(v) \int_{0}^{1} c_{n}(u) \times
$$$$
\times g_{V}(u, v, w, T) \frac{\partial \tilde{V}_{i-100}(u, v, w, \tau)}{\partial u} d w d v d u d \tau-2 \pi \sqrt{\frac{D_{0 V}}{D_{0 I}}} \sum_{n=1}^{\infty} n c_{n}(\chi) c_{n}(\eta) c_{n}(\phi) e_{n V}(\vartheta) \times
$$$$
\times \int_{0}^{\vartheta} e_{n V}(-\tau) \int_{0}^{1} c_{n}(u) \int_{0}^{1} s_{n}(v) \int_{0}^{1} c_{n}(u) g_{V}(u, v, w, T) \frac{\partial \tilde{V}_{i-100}(u, v, w, \tau)}{\partial v} d w d v d u d \tau-2 \pi \sqrt{\frac{D_{0 V}}{D_{0 I}}} \times
$$ 


$$
\begin{aligned}
& \times \sum_{n=1}^{\infty} n e_{n V}(\vartheta) \int_{0}^{\vartheta} e_{n V}(-\tau) \int_{0}^{1} c_{n}(u) \int_{0}^{1} c_{n}(v) \int_{0}^{1} s_{n}(u) g_{V}(u, v, w, T) \frac{\partial \tilde{V}_{i-100}(u, v, w, \tau)}{\partial w} d w d v d u d \tau \times \\
& \times c_{n}(\chi) c_{n}(\eta) c_{n}(\phi)-2 \sum_{n=1}^{\infty} c_{n}(\chi) c_{n}(\eta) c_{n}(\phi) e_{n I}(\vartheta) \int_{0}^{\vartheta} e_{n V}(-\tau) \int_{0}^{1} c_{n}(u) \int_{0}^{1} c_{n}(v) \int_{0}^{1}\left[\tilde{I}_{100}(u, v, w, \tau) \times\right. \\
& \left.\times \tilde{V}_{000}(u, v, w, \tau)+\tilde{I}_{000}(u, v, w, \tau) \tilde{V}_{100}(u, v, w, \tau)\right]\left[1+\varepsilon_{I, V} g_{I, V}(u, v, w, T)\right] c_{n}(v) d w d v d u d \tau ; \\
& \tilde{I}_{101}(\chi, \eta, \phi, \vartheta)=-2 \pi \sqrt{\frac{D_{0 I}}{D_{0 V}}} \sum_{n=1}^{\infty} n c_{n}(\chi) c_{n}(\eta) c_{n}(\phi) e_{n I}(\vartheta) \int_{0}^{\vartheta} e_{n I}(-\tau) \int_{0}^{1} s_{n}(u) \int_{0}^{1} c_{n}(v) \int_{0}^{1} c_{n}(w) \times \\
& \times g_{I}(u, v, w, T) \frac{\partial \tilde{I}_{001}(u, v, w, \tau)}{\partial u} d w d v d u d \tau-2 \pi \sqrt{\frac{D_{0 I}}{D_{0 V}}} \sum_{n=1}^{\infty} n c_{n}(\chi) c_{n}(\eta) c_{n}(\phi) e_{n I}(\vartheta) \times \\
& \times \int_{0}^{\vartheta} e_{n I}(-\tau) \int_{0}^{1} c_{n}(u) \int_{0}^{1} s_{n}(v) \int_{0}^{1} c_{n}(w) g_{I}(u, v, w, T) \frac{\partial \tilde{I}_{001}(u, v, w, \tau)}{\partial v} d w d v d u d \tau-2 \pi \sqrt{\frac{D_{0 I}}{D_{0 V}}} \times \\
& \times \sum_{n=1}^{\infty} n e_{n I}(\vartheta) \int_{0}^{\vartheta} e_{n I}(-\tau) \int_{0}^{1} c_{n}(u) \int_{0}^{1} c_{n}(v) \int_{0}^{1} s_{n}(w) g_{I}(u, v, w, T) \frac{\partial \tilde{I}_{001}(u, v, w, \tau)}{\partial w} d w d v d u d \tau \times \\
& \times c_{n}(\chi) c_{n}(\eta) c_{n}(\phi)-2 \sum_{n=1}^{\infty} c_{n}(\chi) c_{n}(\eta) c_{n}(\phi) e_{n I}(\vartheta) \int_{0}^{\vartheta} e_{n I}(-\tau) \int_{0}^{1}\left[1+\varepsilon_{I, V} g_{I, V}(u, v, w, T)\right] \times \\
& \times c_{n}(u) \tilde{I}_{100}(u, v, w, \tau) \tilde{V}_{000}(u, v, w, \tau) d w d v d u d \tau \\
& \tilde{V}_{101}(\chi, \eta, \phi, \vartheta)=-2 \pi \sqrt{\frac{D_{0 V}}{D_{0 I}}} \sum_{n=1}^{\infty} n c_{n}(\chi) c_{n}(\eta) c_{n}(\phi) e_{n V}(\vartheta) \int_{0}^{\vartheta} e_{n V}(-\tau) \int_{0}^{1} s_{n}(u) \int_{0}^{1} c_{n}(v) \int_{0}^{1} c_{n}(w) \times \\
& \times g_{V}(u, v, w, T) \frac{\partial \tilde{V}_{001}(u, v, w, \tau)}{\partial u} d w d v d u d \tau-2 \pi \sqrt{\frac{D_{0 V}}{D_{0 I}}} \sum_{n=1}^{\infty} n c_{n}(\chi) c_{n}(\eta) c_{n}(\phi) e_{n V}(\vartheta) \times \\
& \times \int_{0}^{\vartheta} e_{n V}(-\tau) \int_{0}^{1} c_{n}(u) \int_{0}^{1} s_{n}(v) \int_{0}^{1} c_{n}(w) g_{V}(u, v, w, T) \frac{\partial \tilde{V}_{001}(u, v, w, \tau)}{\partial v} d w d v d u d \tau-2 \pi \sqrt{\frac{D_{0 V}}{D_{0 I}}} \times \\
& \times \sum_{n=1}^{\infty} n e_{n V}(\vartheta) \int_{0}^{\vartheta} e_{n V}(-\tau) \int_{0}^{1} c_{n}(u) \int_{0}^{1} c_{n}(v) \int_{0}^{1} s_{n}(w) g_{V}(u, v, w, T) \frac{\partial \tilde{V}_{001}(u, v, w, \tau)}{\partial w} d w d v d u d \tau \times \\
& \times c_{n}(\chi) c_{n}(\eta) c_{n}(\phi)-2 \sum_{n=1}^{\infty} c_{n}(\chi) c_{n}(\eta) c_{n}(\phi) e_{n V}(\vartheta) \int_{0}^{\vartheta} e_{n V}(-\tau) \int_{0}^{1} c_{n}(u) \int_{0}^{1} c_{n}(v) \int_{0}^{1} \tilde{I}_{000}(u, v, w, \tau) \times \\
& \times c_{n}(w)\left[1+\varepsilon_{I, V} g_{I, V}(u, v, w, T)\right] \tilde{V}_{100}(u, v, w, \tau) d w d v d u d \tau ; \\
& \tilde{I}_{011}(\chi, \eta, \phi, \vartheta)=-2 \sum_{n=1}^{\infty} c_{n}(\chi) c_{n}(\eta) c_{n}(\phi) e_{n I}(\vartheta) \int_{0}^{\vartheta} e_{n I}(-\tau) \int_{0}^{1} c_{n}(u) \int_{0}^{1} c_{n}(v) \int_{0}^{1} c_{n}(w)\left\{\left[1+\varepsilon_{I, I} g_{I, I}(u, v, w, T)\right] \times\right.
\end{aligned}
$$$$
\left.\times \tilde{I}_{000}(u, v, w, \tau) \tilde{I}_{010}(u, v, w, \tau)+\left[1+\varepsilon_{I, V} g_{I, V}(u, v, w, T)\right] \tilde{I}_{001}(u, v, w, \tau) \tilde{V}_{000}(u, v, w, \tau)\right\} d w d v d u d \tau
$$$$
\tilde{V}_{011}(\chi, \eta, \phi, \vartheta)=-2 \sum_{n=1}^{\infty} c_{n}(\chi) c_{n}(\eta) c_{n}(\phi) e_{n V}(\vartheta) \int_{0}^{\vartheta} e_{n V}(-\tau) \int_{0}^{1} c_{n}(u) \int_{0}^{1} c_{n}(v) \int_{0}^{1} c_{n}(w)\left\{\left[1+\varepsilon_{V, V} g_{V, V}(u, v, w, T)\right] \times\right.
$$$$
\left.\times \tilde{V}_{000}(u, v, w, \tau) \tilde{V}_{010}(u, v, w, \tau)+\left[1+\varepsilon_{I, V} g_{I, V}(u, v, w, T)\right] \tilde{I}_{000}(u, v, w, \tau) \tilde{V}_{001}(u, v, w, \tau)\right\} d w d v d u d \tau .
$$

Equations for initial-order approximation of concentrations of simplest complexes of radiation defects $\Phi_{\rho 0}(x, y, z, t)$, corrections for the approximation $\Phi_{\rho i}(x, y, z, t)(i \geq 1)$, boundary and initial conditions for them have been obtain by substitution of the series (14) into Eqs.(6) and conditions (7) and equating of coefficients at equal powers of parameters, which have been used in the series (14). The equations and conditions could be written as 


$$
\begin{aligned}
& \frac{\partial \Phi_{I 0}(x, y, z, t)}{\partial t}=D_{0 \Phi I} \frac{\partial^{2} \Phi_{I 0}(x, y, z, t)}{\partial x^{2}}+D_{0 \Phi I} \frac{\partial^{2} \Phi_{I 0}(x, y, z, t)}{\partial y^{2}}+D_{0 \Phi I} \frac{\partial^{2} \Phi_{I 0}(x, y, z, t)}{\partial z^{2}}+ \\
& +k_{I, I}(x, y, z, T) I^{2}(x, y, z, t)-k_{I}(x, y, z, T) I(x, y, z, t) \\
& \frac{\partial \Phi_{V 0}(x, y, z, t)}{\partial t}=D_{0 \Phi V} \frac{\partial^{2} \Phi_{V 0}(x, y, z, t)}{\partial x^{2}}+D_{0 \Phi V} \frac{\partial^{2} \Phi_{V 0}(x, y, z, t)}{\partial y^{2}}+D_{0 \Phi V} \frac{\partial^{2} \Phi_{V 0}(x, y, z, t)}{\partial z^{2}}+ \\
& +k_{V, V}(x, y, z, T) V^{2}(x, y, z, t)-k_{V}(x, y, z, T) V(x, y, z, t) ; \\
& \frac{\partial \Phi_{I i}(x, y, z, t)}{\partial t}=D_{0 \Phi I} \frac{\partial^{2} \Phi_{I i}(x, y, z, t)}{\partial x^{2}}+D_{0 \Phi I} \frac{\partial^{2} \Phi_{I i}(x, y, z, t)}{\partial y^{2}}+D_{0 \Phi I} \frac{\partial^{2} \Phi_{I i}(x, y, z, t)}{\partial z^{2}}+ \\
& +D_{0 \Phi I} \frac{\partial}{\partial x}\left[g_{\Phi I}(x, y, z, T) \frac{\partial \Phi_{I i-1}(x, y, z, t)}{\partial x}\right]+D_{0 \Phi I} \frac{\partial}{\partial y}\left[g_{\Phi I}(x, y, z, T) \frac{\partial \Phi_{I i-1}(x, y, z, t)}{\partial y}\right]+ \\
& +D_{0 \Phi I} \frac{\partial}{\partial z}\left[g_{\Phi I}(x, y, z, T) \frac{\partial \Phi_{I i-1}(x, y, z, t)}{\partial z}\right] \\
& \frac{\partial \Phi_{V i}(x, y, z, t)}{\partial t}=D_{0 \Phi V} \frac{\partial^{2} \Phi_{V i}(x, y, z, t)}{\partial x^{2}}+D_{0 \Phi V} \frac{\partial^{2} \Phi_{V i}(x, y, z, t)}{\partial y^{2}}+D_{0 \Phi V} \frac{\partial^{2} \Phi_{V i}(x, y, z, t)}{\partial z^{2}}+ \\
& +D_{0 \Phi V} \frac{\partial}{\partial x}\left[g_{\Phi V}(x, y, z, T) \frac{\partial \Phi_{V i-1}(x, y, z, t)}{\partial x}\right]+D_{0 \Phi V} \frac{\partial}{\partial y}\left[g_{\Phi V}(x, y, z, T) \frac{\partial \Phi_{V i-1}(x, y, z, t)}{\partial y}\right]+ \\
& +D_{0 \Phi V} \frac{\partial}{\partial z}\left[g_{\Phi V}(x, y, z, T) \frac{\partial \Phi_{V i-1}(x, y, z, t)}{\partial z}\right], i \geq 1 \\
& \left.\frac{\partial \Phi_{\rho i}(x, y, z, t)}{\partial x}\right|_{x=0}=0,\left.\frac{\partial \Phi_{\rho i}(x, y, z, t)}{\partial x}\right|_{x=L_{x}}=0,\left.\frac{\partial \Phi_{\rho i}(x, y, z, t)}{\partial y}\right|_{y=0}=0,\left.\frac{\partial \Phi_{\rho i}(x, y, z, t)}{\partial y}\right|_{y=L_{y}}=0, \\
& \left.\frac{\partial \Phi_{\rho i}(x, y, z, t)}{\partial z}\right|_{z=0}=0,\left.\frac{\partial \Phi_{\rho i}(x, y, z, t)}{\partial z}\right|_{z=L_{z}}=0, i>0 ; \Phi_{\rho 0}(x, y, z, 0)=f_{\Phi \rho}(x, y, z), \Phi_{\rho i}(x, y, z, 0)=0, i>1 .
\end{aligned}
$$

Solutions of the above equations with account boundary and initial conditions for them have been calculated by using standard Fourier approach $[31,32]$. Using the approach leads to the following result

$$
\begin{gathered}
\Phi_{\rho 0}(x, y, z, t)=\frac{1}{L_{x} L_{y} L_{z}}+\frac{2}{L_{x} L_{y} L_{z}} \sum_{n=1}^{\infty} F_{n \Phi_{\rho}} c_{n}(x) c_{n}(y) c_{n}(z) e_{n \Phi}(t)+\frac{2}{L} \sum_{n=1}^{\infty} n c_{n}(x) c_{n}(y) c_{n}(z) \times \\
\times e_{\Phi_{\rho} n}(t) \int_{0}^{t} e_{\Phi_{\rho^{n}}}(-\tau) \int_{0}^{L_{x}} c_{n}(u) \int_{0}^{L_{y}} c_{n}(v) \int_{0}^{L_{z}}\left[k_{I, I}(u, v, w, T) I^{2}(u, v, w, \tau)-k_{I}(u, v, w, T) I(u, v, w, \tau)\right] \times \\
\times c_{n}(w) d w d v d u d \tau,
\end{gathered}
$$

where $e_{n \Phi_{\rho}}(t)=\exp \left[-\pi^{2} n^{2} D_{0 \Phi_{\rho}} t\left(\frac{1}{L_{x}^{2}}+\frac{1}{L_{y}^{2}}+\frac{1}{L_{z}^{2}}\right)\right], F_{n \Phi_{\rho}}=\int_{0}^{L_{x}} c_{n}(u) \int_{0}^{L_{y}} c_{n}(v) \int_{0}^{L_{z}} f_{\Phi_{\rho}}(u, v, w) \times$ $\times c_{n}(w) d w d v d u, c_{n}(x)=\cos \left(\pi n x / L_{x}\right)$;

$$
\begin{aligned}
& \Phi_{\rho i}(x, y, z, t)=-\frac{2 \pi}{L_{x}^{2} L_{y} L_{z}} \sum_{n=1}^{\infty} n c_{n}(x) c_{n}(y) c_{n}(z) e_{\Phi_{\rho} n}(t) \int_{0}^{t} e_{\Phi_{\rho} n}(-\tau) \int_{0}^{L_{x}} s_{n}(u) \int_{0}^{L_{y}} c_{n}(v) \int_{0}^{L_{z}} g_{\Phi_{\rho}}(u, v, w, T) \times \\
& \times c_{n}(w) \frac{\partial \Phi_{I_{\rho} i-1}(u, v, w, \tau)}{\partial u} d w d v d u d \tau-\frac{2 \pi}{L_{x} L_{y}^{2} L_{z}} \sum_{n=1}^{\infty} n c_{n}(x) c_{n}(y) c_{n}(z) e_{\Phi_{\rho} n}(t) \int_{0}^{t} e_{\Phi_{\rho} n}(-\tau) \int_{0}^{L_{x}} c_{n}(u) \times
\end{aligned}
$$




$$
\begin{aligned}
& \times \int_{0}^{L_{y}} s_{n}(v) \int_{0}^{L_{z}} c_{n}(w) g_{\Phi_{\rho}}(u, v, w, T) \frac{\partial \Phi_{I_{\rho} i-1}(u, v, w, \tau)}{\partial v} d w d v d u d \tau-\frac{2 \pi}{L_{x} L_{y} L_{z}^{2}} \sum_{n=1}^{\infty} n c_{n}(x) c_{n}(y) e_{\Phi_{\rho} n}(t) \times \\
& \quad \times c_{n}(z) \int_{0}^{t} e_{\Phi_{\rho^{n}}}(-\tau) \int_{0}^{L_{x}} c_{n}(u) \int_{0}^{L_{y}} c_{n}(v) \int_{0}^{L_{z}} s_{n}(w) g_{\Phi_{\rho}}(u, v, w, T) \frac{\partial \Phi_{I_{\rho} i-1}(u, v, w, \tau)}{\partial w} d w d v d u d \tau, i \geq 1,
\end{aligned}
$$

where $s_{n}(x)=\sin \left(\pi n x / L_{x}\right)$.

Equations for initial-order approximation of dopant concentration $C_{00}(x, y, z, t)$, corrections for the approximation $C_{i j}(x, y, z, t)(i \geq 1, j \geq 1)$, boundary and initial conditions for them have been obtain by substitution of the series (15) into Eqs.(1) and conditions (2) and equating of coefficients at equal powers of parameters, which have been used in the series (15). The equations and conditions could be written as

$$
\begin{aligned}
& \frac{\partial C_{00}(x, y, z, t)}{\partial t}=D_{0 L} \frac{\partial^{2} C_{00}(x, y, z, t)}{\partial x^{2}}+D_{0 L} \frac{\partial^{2} C_{00}(x, y, z, t)}{\partial y^{2}}+D_{0 L} \frac{\partial^{2} C_{00}(x, y, z, t)}{\partial z^{2}} \text {; } \\
& \frac{\partial C_{i 0}(x, y, z, t)}{\partial t}=D_{0 L} \frac{\partial^{2} C_{i 0}(x, y, z, t)}{\partial x^{2}}+D_{0 L} \frac{\partial^{2} C_{i 0}(x, y, z, t)}{\partial y^{2}}+D_{0 L} \frac{\partial^{2} C_{i 0}(x, y, z, t)}{\partial z^{2}}+ \\
& +D_{0 L} \frac{\partial}{\partial x}\left[g_{L}(x, y, z, T) \frac{\partial C_{i-10}(x, y, z, t)}{\partial x}\right]+D_{0 L} \frac{\partial}{\partial y}\left[g_{L}(x, y, z, T) \frac{\partial C_{i-10}(x, y, z, t)}{\partial y}\right]+ \\
& +D_{0 L} \frac{\partial}{\partial z}\left[g_{L}(x, y, z, T) \frac{\partial C_{i-10}(x, y, z, t)}{\partial z}\right], i \geq 1 ; \\
& \frac{\partial C_{01}(x, y, z, t)}{\partial t}=D_{0 L} \frac{\partial^{2} C_{01}(x, y, z, t)}{\partial x^{2}}+D_{0 L} \frac{\partial^{2} C_{01}(x, y, z, t)}{\partial y^{2}}+D_{0 L} \frac{\partial^{2} C_{01}(x, y, z, t)}{\partial z^{2}}+ \\
& +D_{0 L} \frac{\partial}{\partial x}\left[\frac{C_{00}^{\gamma}(x, y, z, t)}{P^{\gamma}(x, y, z, T)} \frac{\partial C_{00}(x, y, z, t)}{\partial x}\right]+D_{0 L} \frac{\partial}{\partial y}\left[\frac{C_{00}^{\gamma}(x, y, z, t)}{P^{\gamma}(x, y, z, T)} \frac{\partial C_{00}(x, y, z, t)}{\partial y}\right]+ \\
& +D_{0 L} \frac{\partial}{\partial z}\left[\frac{C_{00}^{\gamma}(x, y, z, t)}{P^{\gamma}(x, y, z, T)} \frac{\partial C_{00}(x, y, z, t)}{\partial z}\right] \\
& \frac{\partial C_{02}(x, y, z, t)}{\partial t}=D_{0 L} \frac{\partial^{2} C_{02}(x, y, z, t)}{\partial x^{2}}+D_{0 L} \frac{\partial^{2} C_{02}(x, y, z, t)}{\partial y^{2}}+D_{0 L} \frac{\partial^{2} C_{02}(x, y, z, t)}{\partial z^{2}}+ \\
& +D_{0 L} \frac{\partial}{\partial x}\left[C_{01}(x, y, z, t) \frac{C_{00}^{\gamma-1}(x, y, z, t)}{P^{\gamma}(x, y, z, T)} \frac{\partial C_{00}(x, y, z, t)}{\partial x}\right]+\frac{\partial}{\partial y}\left[C_{01}(x, y, z, t) \frac{C_{00}^{\gamma-1}(x, y, z, t)}{P^{\gamma}(x, y, z, T)} \times\right. \\
& \left.\times \frac{\partial C_{00}(x, y, z, t)}{\partial y}\right] D_{0 L}+D_{0 L} \frac{\partial}{\partial z}\left[C_{01}(x, y, z, t) \frac{C_{00}^{\gamma-1}(x, y, z, t)}{P^{\gamma}(x, y, z, T)} \frac{\partial C_{00}(x, y, z, t)}{\partial z}\right]+D_{0 L} \times \\
& \times \frac{\partial}{\partial x}\left[\frac{C_{00}^{\gamma}(x, y, z, t)}{P^{\gamma}(x, y, z, T)} \frac{\partial C_{01}(x, y, z, t)}{\partial x}\right]+D_{0 L} \frac{\partial}{\partial y}\left[\frac{C_{00}^{\gamma}(x, y, z, t)}{P^{\gamma}(x, y, z, T)} \frac{\partial C_{01}(x, y, z, t)}{\partial y}\right]+ \\
& +D_{0 L} \frac{\partial}{\partial z}\left[\frac{C_{00}^{\gamma}(x, y, z, t)}{P^{\gamma}(x, y, z, T)} \frac{\partial C_{01}(x, y, z, t)}{\partial z}\right] \\
& \frac{\partial C_{11}(x, y, z, t)}{\partial t}=D_{0 L} \frac{\partial^{2} C_{11}(x, y, z, t)}{\partial x^{2}}+D_{0 L} \frac{\partial^{2} C_{11}(x, y, z, t)}{\partial y^{2}}+D_{0 L} \frac{\partial^{2} C_{11}(x, y, z, t)}{\partial z^{2}}+
\end{aligned}
$$


International Journal of Recent advances in Physics (IJRAP) Vol.4, No.2, May 2015

$$
\begin{gathered}
+D_{0 L} \frac{\partial}{\partial x}\left[C_{10}(x, y, z, t) \frac{C_{00}^{\gamma-1}(x, y, z, t)}{P^{\gamma}(x, y, z, T)} \frac{\partial C_{00}(x, y, z, t)}{\partial x}\right]+\frac{\partial}{\partial y}\left[C_{10}(x, y, z, t) \frac{C_{00}^{\gamma-1}(x, y, z, t)}{P^{\gamma}(x, y, z, T)} \times\right. \\
\left.\times \frac{\partial C_{00}(x, y, z, t)}{\partial y}\right] D_{0 L}+D_{0 L} \frac{\partial}{\partial z}\left[C_{10}(x, y, z, t) \frac{C_{00}^{\gamma-1}(x, y, z, t)}{P^{\gamma}(x, y, z, T)} \frac{\partial C_{00}(x, y, z, t)}{\partial z}\right]+D_{0 L} \times \\
\times \frac{\partial}{\partial x}\left[\frac{C_{00}^{\gamma}(x, y, z, t)}{P^{\gamma}(x, y, z, T)} \frac{\partial C_{10}(x, y, z, t)}{\partial x}\right]+D_{0 L} \frac{\partial}{\partial y}\left[\frac{C_{00}^{\gamma}(x, y, z, t)}{P^{\gamma}(x, y, z, T)} \frac{\partial C_{10}(x, y, z, t)}{\partial y}\right]+ \\
+D_{0 L} \frac{\partial}{\partial z}\left[\frac{C_{00}^{\gamma}(x, y, z, t)}{P^{\gamma}(x, y, z, T)} \frac{\partial C_{10}(x, y, z, t)}{\partial z}\right]+D_{0 L} \frac{\partial}{\partial x}\left[g_{L}(x, y, z, T) \frac{\partial C_{01}(x, y, z, t)}{\partial x}\right]+ \\
+D_{0 L} \frac{\partial}{\partial y}\left[g_{L}(x, y, z, T) \frac{\partial C_{01}(x, y, z, t)}{\partial y}\right]+D_{0 L} \frac{\partial}{\partial z}\left[g_{L}(x, y, z, T) \frac{\partial C_{01}(x, y, z, t)}{\partial z}\right] ; \\
\left.\frac{\partial C_{i j}(x, y, z, t)}{\partial x}\right|_{x=0}=0,\left.\frac{\partial C_{i j}(x, y, z, t)}{\partial x}\right|_{x=L_{x}}=0,\left.\frac{\partial C_{i j}(x, y, z, t)}{\partial y}\right|_{y=0}=0,\left.\frac{\partial C_{i j}(x, y, z, t)}{\partial y}\right|_{y=L_{y}}=0, \\
\quad \frac{\left.\partial C_{i j}(x, y, z, t)\right|_{z=0}=0,\left.\frac{\partial C_{i j}(x, y, z, t)}{\partial z}\right|_{z=L_{z}}=0, i \geq 0, j \geq 0 ;}{\partial z}=1, j \geq 1 .
\end{gathered}
$$

Solutions of the above equations with account boundary and initial conditions for them have been calculated by using standard Fourier approach $[31,32]$. Using the approach leads to the following result

$$
C_{00}(x, y, z, t)=\frac{1}{L_{x} L_{y} L_{z}}+\frac{2}{L_{x} L_{y} L_{z}} \sum_{n=1}^{\infty} F_{n C} c_{n}(x) c_{n}(y) c_{n}(z) e_{n C}(t),
$$

where $e_{n \Phi_{\rho}}(t)=\exp \left[-\pi^{2} n^{2} D_{0 \Phi_{\rho}} t\left(\frac{1}{L_{x}^{2}}+\frac{1}{L_{y}^{2}}+\frac{1}{L_{z}^{2}}\right)\right], F_{n C}=\int_{0}^{L_{x}} c_{n}(u) \int_{0}^{L_{y}} c_{n}(v) \int_{0}^{L_{z}} f_{C}(u, v, w) \times$ $\times c_{n}(w) d w d v d u$

$$
\begin{aligned}
& C_{i 0}(x, y, z, t)=-\frac{2 \pi}{L_{x}^{2} L_{y} L_{z}} \sum_{n=1}^{\infty} n F_{n C} c_{n}(x) c_{n}(y) c_{n}(z) e_{n C}(t) \int_{0}^{t} e_{n C}(-\tau) \int_{0}^{L_{x}} s_{n}(u) \int_{0}^{L_{y}} c_{n}(v) \int_{0}^{L_{z}} g_{L}(u, v, w, T) \times \\
& \times c_{n}(v) \frac{\partial C_{i-10}(u, v, w, \tau)}{\partial u} d w d v d u d \tau-\frac{2 \pi}{L_{x} L_{y}^{2} L_{z}} \sum_{n=1}^{\infty} n F_{n C} c_{n}(x) c_{n}(y) c_{n}(z) e_{n C}(t) \int_{0}^{t} e_{n C}(-\tau) \int_{0}^{L_{x}} c_{n}(u) \times \\
& \times \int_{0}^{L_{y}} s_{n}(v) \int_{0}^{L_{z}} c_{n}(v) g_{L}(u, v, w, T) \frac{\partial C_{i-10}(u, v, w, \tau)}{\partial v} d w d v d u d \tau-\frac{2 \pi}{L_{x} L_{y} L_{z}^{2}} \sum_{n=1}^{\infty} n F_{n C} c_{n}(x) c_{n}(y) c_{n}(z) \times \\
& \times e_{n C}(t) \int_{0}^{t} e_{n C}(-\tau) \int_{0}^{L_{x}} c_{n}(u) \int_{0}^{L_{y}} c_{n}(v) \int_{0}^{L_{z}} s_{n}(v) g_{L}(u, v, w, T) \frac{\partial C_{i-10}(u, v, w, \tau)}{\partial w} d w d v d u d \tau, i \geq 1 ; \\
& C_{01}(x, y, z, t)=-\frac{2 \pi}{L_{x}^{2} L_{y} L_{z}} \sum_{n=1}^{\infty} n F_{n C} c_{n}(x) c_{n}(y) c_{n}(z) e_{n C}(t) \int_{0}^{t} e_{n C}(-\tau) \int_{0}^{L_{x}} s_{n}(u) \int_{0}^{L_{y}} c_{n}(v) \int_{0}^{L_{z}} \frac{C_{00}^{\gamma}(u, v, w, \tau)}{P^{\gamma}(u, v, w, T)} \times \\
& \times c_{n}(w) \frac{\partial C_{00}(u, v, w, \tau)}{\partial u} d w d v d u d \tau-\frac{2 \pi}{L_{x} L_{y}^{2} L_{z}} \sum_{n=1}^{\infty} n F_{n C} c_{n}(x) c_{n}(y) c_{n}(z) e_{n C}(t) \int_{0}^{t} e_{n C}(-\tau) \int_{0}^{L_{x}} c_{n}(u) \times \\
& \times \int_{0}^{L_{y}} s_{n}(v) \int_{0}^{L_{z}} c_{n}(w) \frac{C_{00}^{\gamma}(u, v, w, \tau)}{P^{\gamma}(u, v, w, T)} \frac{\partial C_{00}(u, v, w, \tau)}{\partial v} d w d v d u d \tau-\frac{2 \pi}{L_{x} L_{y} L_{z}^{2}} \sum_{n=1}^{\infty} n F_{n C} c_{n}(x) c_{n}(y) c_{n}(z) \times
\end{aligned}
$$




$$
\begin{aligned}
& \times e_{n C}(t) \int_{0}^{t} e_{n C}(-\tau) \int_{0}^{L_{x}} c_{n}(u) \int_{0}^{L_{y}} c_{n}(v) \int_{0}^{L_{z}} s_{n}(w) \frac{C_{00}^{\gamma}(u, v, w, \tau)}{P^{\gamma}(u, v, w, T)} \frac{\partial C_{00}(u, v, w, \tau)}{\partial w} d w d v d u d \tau \\
& C_{02}(x, y, z, t)=-\frac{2 \pi}{L_{x}^{2} L_{y} L_{z}} \sum_{n=1}^{\infty} n F_{n C} c_{n}(x) c_{n}(y) c_{n}(z) e_{n C}(t) \int_{0}^{t} e_{n C}(-\tau) \int_{0}^{L_{x}} s_{n}(u) \int_{0}^{L_{y}} c_{n}(v) \int_{0}^{L_{z}} c_{n}(w) \times \\
& \times C_{01}(u, v, w, \tau) \frac{C_{00}^{\gamma-1}(u, v, w, \tau)}{P^{\gamma}(u, v, w, T)} \frac{\partial C_{00}(u, v, w, \tau)}{\partial u} d w d v d u d \tau-\frac{2 \pi}{L_{x} L_{y}^{2} L_{z}} \sum_{n=1}^{\infty} F_{n C} c_{n}(x) c_{n}(y) \times \\
& \times n c_{n}(z) e_{n C}(t) \int_{0}^{t} e_{n C}(-\tau) \int_{0}^{L_{x}} c_{n}(u) \int_{0}^{L_{y}} s_{n}(v) \int_{0}^{L_{z}} C_{01}(u, v, w, \tau) \frac{C_{00}^{\gamma-1}(u, v, w, \tau)}{P^{\gamma}(u, v, w, T)} \frac{\partial C_{00}(u, v, w, \tau)}{\partial v} \times \\
& \times c_{n}(w) d w d v d u d \tau-\frac{2 \pi}{L_{x} L_{y} L_{z}^{2}} \sum_{n=1}^{\infty} n F_{n C} c_{n}(x) c_{n}(y) c_{n}(z) e_{n C}(t) \int_{0}^{t} e_{n C}(-\tau) \int_{0}^{L_{x}} c_{n}(u) \int_{0}^{L_{y}} c_{n}(v) \times \\
& \times \int_{0}^{L_{z}} s_{n}(w) C_{01}(u, v, w, \tau) \frac{C_{00}^{\gamma-1}(u, v, w, \tau)}{P^{\gamma}(u, v, w, T)} \frac{\partial C_{00}(u, v, w, \tau)}{\partial w} d w d v d u d \tau-\frac{2 \pi}{L_{x}^{2} L_{y} L_{z}} \sum_{n=1}^{\infty} n c_{n}(x) \times \\
& \times c_{n}(y) c_{n}(z) e_{n C}(t) \int_{0}^{t} e_{n C}(-\tau) \int_{0}^{L_{x}} s_{n}(u) \int_{0}^{L_{y}} c_{n}(v) \int_{0}^{L_{z}} C_{01}(u, v, w, \tau) \frac{C_{00}^{\gamma-1}(u, v, w, \tau)}{P^{\gamma}(u, v, w, T)} \frac{\partial C_{00}(u, v, w, \tau)}{\partial u} \times \\
& \times F_{n C} c_{n}(w) \frac{\partial C_{00}(u, v, w, \tau)}{\partial u} d w d v d u d \tau-\frac{2 \pi}{L_{x} L_{y}^{2} L_{z}} \sum_{n=1}^{\infty} n c_{n}(x) c_{n}(y) c_{n}(z) e_{n C}(t) \int_{0}^{t} e_{n C}(-\tau) \times \\
& \times F_{n C} \int_{0}^{L_{x}} c_{n}(u) \int_{0}^{L_{y}} s_{n}(v) \int_{0}^{L_{z}} c_{n}(w) C_{01}(u, v, w, \tau) \frac{C_{00}^{\gamma-1}(u, v, w, \tau)}{P^{\gamma}(u, v, w, T)} \frac{\partial C_{00}(u, v, w, \tau)}{\partial v} d w d v d u d \tau- \\
& -\frac{2 \pi}{L_{x} L_{y} L_{z}^{2}} \sum_{n=1}^{\infty} n F_{n C} c_{n}(x) c_{n}(y) c_{n}(z) e_{n C}(t) \int_{0}^{t} e_{n C}(-\tau) \int_{0}^{L_{x}} c_{n}(u) \int_{0}^{L_{y}} c_{n}(v) \int_{0}^{L_{z}} s_{n}(w) C_{01}(u, v, w, \tau) \times \\
& \times \frac{C_{00}^{\gamma-1}(u, v, w, \tau)}{P^{\gamma}(u, v, w, T)} \frac{\partial C_{00}(u, v, w, \tau)}{\partial w} d w d v d u d \tau-\frac{2 \pi}{L_{x}^{2} L_{y} L_{z}} \sum_{n=1}^{\infty} n c_{n}(x) c_{n}(y) c_{n}(z) e_{n C}(t) \times \\
& \times F_{n C} \int_{0}^{t} e_{n C}(-\tau) \int_{0}^{L_{x}} s_{n}(u) \int_{0}^{L_{y}} c_{n}(v) \int_{0}^{L_{z}} c_{n}(w) \frac{C_{00}^{\gamma}(u, v, w, \tau)}{P^{\gamma}(u, v, w, T)} \frac{\partial C_{01}(u, v, w, \tau)}{\partial u} d w d v d u d \tau-\frac{2 \pi}{L_{x} L_{y}^{2}} \times \\
& \times \frac{1}{L_{z}} \sum_{n=1}^{\infty} e_{n C}(t) \int_{0}^{t} e_{n C}(-\tau) \int_{0}^{L_{x}} c_{n}(u) \int_{0}^{L_{y}} s_{n}(v) \int_{0}^{L_{z}} c_{n}(w) \frac{C_{00}^{\gamma}(u, v, w, \tau)}{P^{\gamma}(u, v, w, T)} \frac{\partial C_{01}(u, v, w, \tau)}{\partial v} d w d v d u d \tau \times \\
& \times n F_{n C} c_{n}(x) c_{n}(y) c_{n}(z)-\frac{2 \pi}{L_{x} L_{y} L_{z}^{2}} \sum_{n=1}^{\infty} n F_{n C} c_{n}(x) c_{n}(y) c_{n}(z) e_{n C}(t) \int_{0}^{t} e_{n C}(-\tau) \int_{0}^{L_{x}} c_{n}(u) \int_{0}^{L_{y}} c_{n}(v) \times \\
& \times \int_{0}^{L_{z}} s_{n}(w) \frac{C_{00}^{\gamma}(u, v, w, \tau)}{P^{\gamma}(u, v, w, T)} \frac{\partial C_{01}(u, v, w, \tau)}{\partial w} d w d v d u d \tau \\
& C_{11}(x, y, z, t)=-\frac{2 \pi}{L_{x}^{2} L_{y} L_{z}} \sum_{n=1}^{\infty} n F_{n C} c_{n}(x) c_{n}(y) c_{n}(z) e_{n C}(t) \int_{0}^{t} e_{n C}(-\tau) \int_{0}^{L_{x}} s_{n}(u) \int_{0}^{L_{y}} c_{n}(v) \int_{0}^{L_{z}} c_{n}(w) \times \\
& \times g_{L}(u, v, w, T) \frac{\partial C_{01}(u, v, w, \tau)}{\partial u} d w d v d u d \tau-\frac{2 \pi}{L_{x} L_{y}^{2} L_{z}} \sum_{n=1}^{\infty} n F_{n C} c_{n}(x) c_{n}(y) c_{n}(z) e_{n C}(t) \times \\
& \times \int_{0}^{t} e_{n C}(-\tau) \int_{0}^{L_{x}} c_{n}(u) \int_{0}^{L_{y}} s_{n}(v) \int_{0}^{L_{z}} c_{n}(w) g_{L}(u, v, w, T) \frac{\partial C_{01}(u, v, w, \tau)}{\partial v} d w d v d u d \tau-\frac{2 \pi}{L_{x} L_{y} L_{z}^{2}} \times \\
& \times \sum_{n=1}^{\infty} n F_{n C} c_{n}(x) c_{n}(y) c_{n}(z) e_{n C}(t) \int_{0}^{t} e_{n C}(-\tau) \int_{0}^{L_{x}} c_{n}(u) \int_{0}^{L_{y}} c_{n}(v) \int_{0}^{L_{z}} g_{L}(u, v, w, T) \frac{\partial C_{01}(u, v, w, \tau)}{\partial w} \times
\end{aligned}
$$




$$
\begin{aligned}
& \times s_{n}(w) d w d v d u d \tau-\frac{2 \pi}{L_{x}^{2} L_{y} L_{z}} \sum_{n=1}^{\infty} n F_{n C} c_{n}(x) c_{n}(y) c_{n}(z) e_{n C}(t) \int_{0}^{t} e_{n C}(-\tau) \int_{0}^{L_{x}} s_{n}(u) \int_{0}^{L_{y}} c_{n}(v) \times \\
& \times \int_{0}^{L_{z}} c_{n}(w) \frac{C_{00}^{\gamma}(u, v, w, \tau)}{P^{\gamma}(u, v, w, T)} \frac{\partial C_{10}(u, v, w, \tau)}{\partial u} d w d v d u d \tau-\frac{2 \pi}{L_{x} L_{y}^{2} L_{z}} \sum_{n=1}^{\infty} n F_{n C} c_{n}(x) c_{n}(y) c_{n}(z) \times \\
& \times e_{n C}(t) \int_{0}^{t} e_{n C}(-\tau) \int_{0}^{L_{x}} c_{n}(u) \int_{0}^{L_{y}} s_{n}(v) \int_{0}^{L_{z}} c_{n}(w) \frac{C_{00}^{\gamma}(u, v, w, \tau)}{P^{\gamma}(u, v, w, T)} \frac{\partial C_{10}(u, v, w, \tau)}{\partial v} d w d v d u d \tau-\frac{2}{L_{x}} \times \\
& \times \sum_{n=1}^{\infty} n F_{n C} c_{n}(x) c_{n}(y) c_{n}(z) e_{n C}(t) \int_{0}^{t} e_{n C}(-\tau) \int_{0}^{L_{x}} c_{n}(u) \int_{0}^{L_{y}} c_{n}(v) \int_{0}^{L_{z}} \frac{C_{00}^{\gamma}(u, v, w, \tau)}{P^{\gamma}(u, v, w, T)} \frac{\partial C_{10}(u, v, w, \tau)}{\partial w} \times \\
& \times s_{n}(w) d w d v d u d \tau \frac{\pi}{L_{y} L_{z}^{2}}-\frac{2 \pi}{L_{x}^{2} L_{y} L_{z}} \sum_{n=1}^{\infty} n F_{n C} c_{n}(x) c_{n}(y) c_{n}(z) e_{n C}(t) \int_{0}^{t} e_{n C}(-\tau) \int_{0}^{L_{x}} s_{n}(u) \times \\
& \times \int_{0}^{L_{y}} c_{n}(v) \int_{0}^{L_{z}} c_{n}(w) C_{10}(u, v, w, \tau) \frac{C_{00}^{\gamma-1}(u, v, w, \tau)}{P^{\gamma}(u, v, w, T)} \frac{\partial C_{00}(u, v, w, \tau)}{\partial u} d w d v d u d \tau-\frac{2 \pi}{L_{x} L_{y}^{2} L_{z}} \times \\
& \times \sum_{n=1}^{\infty} n c_{n}(x) c_{n}(y) c_{n}(z) e_{n C}(t) \int_{0}^{t} e_{n C}(-\tau) \int_{0}^{L_{x}} c_{n}(u) \int_{0}^{L_{y}} s_{n}(v) \int_{0}^{L_{z}} \frac{C_{00}^{\gamma-1}(u, v, w, \tau)}{P^{\gamma}(u, v, w, T)} \frac{\partial C_{00}(u, v, w, \tau)}{\partial v} \times \\
& \quad \times c_{n}(w) C_{10}(u, v, w, \tau) d w d v d u d \tau F_{n C}-\frac{2 \pi}{L_{x} L_{y} L_{z}^{2} \sum_{n=1}^{\infty} n F_{n C} c_{n}(x) c_{n}(y) c_{n}(z) e_{n C}(t) \times} \\
& \times \int_{0}^{t} e_{n C}(-\tau) \int_{0}^{L_{x}} c_{n}(u) \int_{0}^{L_{y}} c_{n}(v) \int_{0}^{L_{z}} s_{n}(w) C_{10}(u, v, w, \tau) \frac{C_{00}^{\gamma-1}(u, v, w, \tau)}{P^{\gamma}(u, v, w, T)} \frac{\partial C_{00}(u, v, w, \tau)}{\partial w} d w d v d u d \tau .
\end{aligned}
$$

\section{Authors:}

Pankratov Evgeny Leonidovich was born at 1977. From 1985 to 1995 he was educated in a secondary school in Nizhny Novgorod. From 1995 to 2004 he was educated in Nizhny Novgorod State University: from 1995 to 1999 it was bachelor course in Radiophysics, from 1999 to 2001 it was master course in Radiophysics with specialization in Statistical Radiophysics, from 2001 to 2004 it was PhD course in Radiophysics. From 2004 to 2008 E.L. Pankratov was a leading technologist in Institute for Physics of Microstructures. From 2008 to 2012 E.L. Pankratov was a senior lecture/Associate Professor of Nizhny Novgorod State University of Architecture and Civil Engineering. Now E.L. Pankratov is in his Full Doctor course in Radiophysical Department of Nizhny Novgorod State University. He has 105 published papers in area of his researches.

Bulaeva Elena Alexeevna was born at 1991. From 1997 to 2007 she was educated in secondary school of village Kochunovo of Nizhny Novgorod region. From 2007 to 2009 she was educated in boarding school "Center for gifted children". From 2009 she is a student of Nizhny Novgorod State University of Architecture and Civil Engineering (spatiality "Assessment and management of real estate"). At the same time she is a student of courses "Translator in the field of professional communication" and "Design (interior art)" in the University. E.A. Bulaeva was a contributor of grant of President of Russia (grant № MK-548.2010.2). She has 52 published papers in area of her researches. 